\title{
On analytic flows on the torus which are disjoint from systems of probabilistic origin
}

\author{
by \\ Mariusz Lemańczyk and Magdalena Wysokińska (Toruń)
}

\begin{abstract}
We describe two methods of obtaining analytic flows on the torus which are disjoint from dynamical systems induced by some classical stationary processes.

Introduction. In [9] the notion of ELF-flows has been introduced. These flows are defined as follows: We look at the set of time $t$ automorphisms, $t \in \mathbb{R}$, of an ergodic flow as Markov operators of the underlying $L^{2}$-space, and the flow has the ELF property if the closure of this set in the weak operator topology consists of indecomposable Markov operators. The ELFproperty is automatically satisfied for all mixing systems, however in the weak mixing and non-mixing case it seems to be a common property of systems that might be called of probabilistic origin. Indeed, already in [9] it was remarked that Gaussian systems enjoy the ELF-property. Moreover, in the recent paper [3] it is proved that systems induced by (symmetric) $\alpha$ stable processes as well as Poisson suspension flows are also examples of ELFsystems. This is still generalized in [25] because one of the main consequences of the developed theory of infinitely divisible joinings for systems generated by infinitely divisible stationary processes is that such systems also have the ELF-property.

It is rather clear that the flow induced by a stationary process given by a function of a classical stationary process should also be regarded as one of probabilistic origin. In other words, factors of systems of probabilistic origin are also of probabilistic origin (note that the ELF-property is closed under taking factors). Therefore if we want to exhibit systems different from ELF-flows, it is natural to require that such systems are disjoint (in the sense of Furstenberg [12]) from the ELF-class. We recall that disjoint systems have no nontrivial common factors.
\end{abstract}

2000 Mathematics Subject Classification: 37A10, 37C10.

Key words and phrases: analytic flow on the torus, ELF-flow, disjoint flows, almost analytic cocycle.

Research partially supported by KBN grant 1P03A 03826. 
In the series of papers [9]-[8] some classes of smooth flows on surfaces are shown to be disjoint from ELF-flows. However, no smooth flow appearing in those papers was analytic. In the present paper we will show how to obtain analytic flows on the torus which are disjoint from the ELF-class. Our first method is "generic"-we show that given a positive analytic function (which is not a trigonometric polynomial) on the circle, for a generic set of irrational rotations the resulting special flows are disjoint from all ELF-flows. Our analysis is similar to the one of A. Katok (see Theorem 5.7 in [15]). The second approach uses the a.a.c.c.p. method from [17]. This method has a prescribed set of parameters and, over each irrational $\alpha$ belonging to a certain residual subset of $[0,1)$, it leads to a construction of a real-valued step cocycle $\varphi$ which is cohomologous to an analytic zero mean cocycle. Assuming additionally that $\varphi \in L^{2}$ and $\varphi+C>0$ for a constant $C$, as shown in [9], in order to obtain a special flow (under $\varphi+C$ ) disjoint from the ELF-class we need two conditions to be satisfied: along a certain rigidity time $\left(w_{n}\right)$ for the irrational rotation by $\alpha$,

(A) the sequence of distributions $\left(\left(\varphi^{\left(w_{n}\right)}\right)_{*}\right)_{n \geq 1}$ weakly converges to a non-Dirac measure on $\mathbb{R}$,

(B) the sequence $\left(\left\|\varphi^{\left(w_{n}\right)}\right\|_{L^{2}}\right)_{n \geq 1}$ is bounded.

This is the condition (B) which introduces a new restriction on the set of $\alpha$ 's over which a given a.a.c.c.p. can be realized. We argue however that such a set of $\alpha$ 's is still residual.

It should be noticed that the approach when one deals with limit distributions along a rigidity sequence is not an original one. For example the condition (A) is satisfied for analytic constructions of cocycles over irrational rotations in [28] (see also [22]). Also, convergence towards particular distributions including Gaussian distributions appears in [4]. It might be expected that certain modifications in [28] should also give condition (B), and therefore should also lead to constructions of analytic special flows disjoint from the ELF-class.

Our second approach, which uses the a.a.c.c.p. method, will allow us to construct an uncountable family $\left\{\mathcal{T}^{F_{\varepsilon}}\right\}_{\varepsilon \in E}$ of flows obtained by analytic changes of times for the linear flow on $\mathbb{R}^{2} / \mathbb{Z}^{2}$, i.e. $\mathcal{T}^{F_{\varepsilon}}, \varepsilon \in E$, is determined by

$$
\left\{\begin{array}{l}
\frac{d x}{d t}=\frac{\alpha}{F_{\varepsilon}(x, y)}, \\
\frac{d y}{d t}=\frac{1}{F_{\varepsilon}(x, y)},
\end{array}\right.
$$

such that each flow of the family is disjoint from the ELF-class. In fact, $E=\{0,1\}^{\mathbb{N}}$ and on $E$ we consider the equivalence relation $\sim$ :

$$
\varepsilon \sim \varepsilon^{\prime} \Leftrightarrow \varepsilon_{i}=\varepsilon_{i}^{\prime} \text { eventually. }
$$


The family $\left\{\mathcal{T}^{F_{\varepsilon}}\right\}_{\varepsilon \in E}$ enjoys additionally the property that $\mathcal{T}^{F_{\varepsilon}}$ is disjoint from $\mathcal{T}^{F_{\varepsilon^{\prime}}}$ whenever $\varepsilon \not \varepsilon^{\prime}$. Note that, by the Liouville theorem, each flow $\mathcal{T}^{F_{\varepsilon}}$ preserves a "physical" measure, but this measure depends on $\varepsilon$. By considering the whole family of such flows as non-singular flows for the Lebesgue measure on $\mathbb{R}^{2} / \mathbb{Z}^{2}$ we show that the map $\varepsilon \mapsto \mathcal{T}^{F_{\varepsilon}}$ is Borel, whence we are in the situation which first appeared in [5] (see also e.g. [6]). Consequently, by [5], it is impossible to solve the problem of measure-theoretic classification of flows on $\mathbb{R}^{2} / \mathbb{Z}^{2}$ preserving a "physical" measure by a countable set of Borel invariants (in fact, we prove the same result also for the spectral classification).

By further modifications of the a.a.c.c.p. method we will obtain weakly mixing analytic flows $\left(T_{t}^{f}\right)_{t \in \mathbb{R}}$ such that for every probability Borel measure $P$ on $\mathbb{R}$ the integral Markov operator $\int_{\mathbb{R}} T_{t}^{f} d P(t)$ belongs to the weak closure of the time $t$ automorphisms $T_{t}^{f}, t \in \mathbb{R}$. In particular, using a result of Ageev ([1]), if we denote by $\sigma$ the reduced maximal spectral type of $T=T_{1}^{f}$, then the Gaussian automorphism determined by $\sigma$ will have simple spectrum and in particular, convolutions of the reduced maximal spectral type are pairwise singular (see [15], [24], [27] for similar results on convolutions). Such Gaussian automorphisms are interesting because they have the GAG property while Foiaş-Strătilă's theorem fails for them (see [20]).

The authors would like to thank K. Fraczek for useful discussions on the subject, and the referee for numerous remarks, comments, suggestions and for correcting errors in the original version of the paper.

1. Preliminaries. Throughout the paper we will identify $\mathbb{R} / \mathbb{Z}$ with $[0,1)$ (with addition mod 1 ). Each function defined on $[0,1)$ will be treated as a 1 -periodic function on $\mathbb{R}$. The Lebesgue measure on $[0,1)$ will be denoted by $\mu$. For the complex circle $\{z \in \mathbb{C}:|z|=1\}$ we will use the notation $\mathbb{T}$.

1.1. Continued fraction expansion. We will now recall some basic facts about the continued fraction expansion of an irrational number. Each $\alpha \in$ $(0,1)$ has a representation as a continued fraction

$$
\alpha=\left[0 ; a_{1}, a_{2}, \ldots\right],
$$

where the positive integers $a_{n}$ are called the partial quotients of $\alpha$. This expansion is infinite whenever $\alpha$ is irrational. Put

$$
\begin{aligned}
& q_{0}=1, \quad q_{1}=a_{1}, \quad q_{n+1}=a_{n+1} q_{n}+q_{n-1}, \\
& p_{0}=0, \quad p_{1}=1, \quad p_{n+1}=a_{n+1} p_{n}+p_{n-1} \text {. }
\end{aligned}
$$

The rationals $p_{n} / q_{n}$ are called the convergents of $\alpha$ and the following inequality holds:

$$
\left|\alpha-\frac{p_{n}}{q_{n}}\right|<\frac{1}{q_{n} q_{n+1}}
$$


for $n \geq 0$. Moreover, we have

$$
q_{n+1}\left\|q_{n} \alpha\right\|+q_{n}\left\|q_{n+1} \alpha\right\|=1
$$

where $\|v\|=\operatorname{dist}(v, \mathbb{Z})$ for $v \in \mathbb{R}$. The fractional part of a real number $v$ will be denoted by $\{v\}$. Take now an irrational rotation by $\alpha$ on $X=[0,1)$, $T x=x+\alpha$. Recall some facts (contained e.g. in [17]) about the dynamics of $T$. Fix $n \in \mathbb{N}$ even. Then the union of the disjoint Rokhlin towers

$$
\begin{aligned}
\zeta_{n} & =\left\{\left[0,\left\{q_{n} \alpha\right\}\right), T\left[0,\left\{q_{n} \alpha\right\}\right), \ldots, T^{q_{n+1}-1}\left[0,\left\{q_{n} \alpha\right\}\right)\right\}, \\
\bar{\zeta}_{n} & =\left\{\left[\left\{q_{n+1} \alpha\right\}, 1\right), T\left[\left\{q_{n+1} \alpha\right\}, 1\right), \ldots, T^{q_{n}-1}\left[\left\{q_{n+1} \alpha\right\}, 1\right)\right\}
\end{aligned}
$$

coincides with $[0,1)$. For a subsequence $\left(n_{k}\right) \subset \mathbb{N}$ define

$$
I_{k}=\left[0,\left\{a_{2 n_{k}+1} q_{2 n_{k}} \alpha\right\}\right), \quad J_{t}^{k}=T^{(t-1) q_{2 n_{k}}}\left[0,\left\{q_{2 n_{k}} \alpha\right\}\right)
$$

for $t=1, \ldots, a_{2 n_{k}+1}$. We get

$$
I_{k}=\bigcup_{t=1}^{a_{2 n_{k}+1}} J_{t}^{k},
$$

and for all $t=1, \ldots, a_{2 n_{k}+1}$,

$$
l_{k}:=\left|J_{t}^{k}\right|<\frac{1}{a_{2 n_{k}+1} q_{2 n_{k}}} .
$$

Then $\left\{I_{k}, T I_{k}, \ldots, T^{q_{2 n_{k}}-1} I_{k}\right\}$ is a Rokhlin tower and if we put

$$
\Sigma_{k}=[0,1) \backslash \bigcup_{s=0}^{q_{2 n_{k}}-1} T^{s} I_{k}
$$

then $\Sigma_{k}=\bigcup \bar{\zeta}_{2 n_{k}} \cup \bigcup_{r=q_{2 n_{k}+1}-q_{2 n_{k}-1}}^{q_{2 n_{k}+1}-1} T^{r} J_{1}^{k}$, hence

$$
\mu\left(\Sigma_{k}\right)=q_{2 n_{k}}\left\|q_{2 n_{k}+1} \alpha\right\|+q_{2 n_{k}-1}\left\|q_{2 n_{k}} \alpha\right\|<2 / a_{2 n_{k}+1} .
$$

We will also need the following lemma.

LEMma 1 (see e.g. [17]). Given an infinite increasing sequence $\left(q_{n}\right)$ of natural numbers and a positive real-valued function $r=r\left(q_{n}\right)$, the set

$\left\{\alpha \in[0,1)\right.$ : for infinitely many $n$ we have $\left|\alpha-p_{n} / q_{n}\right|<r\left(q_{n}\right)$, and $p_{n} / q_{n}$ are convergents of $\left.\alpha\right\}$

is residual.

1.2. The class of flows with the ELF-property. Consider a measurable flow $\mathcal{S}=\left(S_{t}\right)_{t \in \mathbb{R}}$, that is, for each $t \in \mathbb{R}, S_{t}$ is an automorphism on a standard probability Borel space $(X, \mathcal{B}, \mu)$ and the corresponding unitary representation of $\mathbb{R}$ on $L^{2}(X, \mathcal{B}, \mu)$ given by $U_{S_{t}}(f)=f \circ S_{t}$ is (weakly) continuous. Depending on the context, $S_{t}$ may denote $U_{S_{t}}$. Assume $\mathcal{S}$ to be ergodic and take another ergodic flow $\mathcal{R}=\left(R_{t}\right)_{t \in \mathbb{R}}$ on $(Y, \mathcal{C}, \nu)$. Now we define a joining of $\mathcal{S}$ and $\mathcal{R}$ as an arbitrary probability measure $\varrho$ on 
$(X \times Y, \mathcal{B} \otimes \mathcal{C})$ which is $\left(S_{t} \times R_{t}\right)_{t \in \mathbb{R}^{-i n v a r i a n t}}$ and whose marginals are $\mu$ and $\nu$ respectively. The set of all joinings between $\mathcal{S}$ and $\mathcal{R}$ will be denoted by $J(\mathcal{S}, \mathcal{R})$. In case $\mathcal{S}=\mathcal{R}$ we write $J(\mathcal{S})$ and speak about self-joinings. The subset of ergodic joinings will be denoted by $J^{\mathrm{e}}(\mathcal{S}, \mathcal{R})\left(J^{\mathrm{e}}(\mathcal{S})\right.$ for ergodic self-joinings).

Having a joining $\varrho \in J(\mathcal{S}, \mathcal{R})$ we define a map $\Phi_{\varrho}: L^{2}(X, \mathcal{B}, \mu) \rightarrow$ $L^{2}(Y, \mathcal{C}, \nu)$ such that for each $f \in L^{2}(X, \mathcal{B}, \mu)$ and $g \in L^{2}(Y, \mathcal{C}, \nu)$,

$$
\int_{Y} \Phi_{\varrho}(f) g d \nu=\int_{X \times Y} f \otimes g d \varrho .
$$

Notice that we get a Markov operator $\Phi_{\varrho}: L^{2}(X, \mathcal{B}, \mu) \rightarrow L^{2}(Y, \mathcal{C}, \nu)$, i.e. a bounded linear operator satisfying

$$
\Phi_{\varrho} 1=\Phi_{\varrho}^{*} 1=1 \quad \text { and } \quad \Phi_{\varrho} f \geq 0 \quad \text { whenever } f \geq 0 .
$$

Conversely, having a Markov operator $\Phi: L^{2}(X, \mathcal{B}, \mu) \rightarrow L^{2}(Y, \mathcal{C}, \nu)$ we can obtain a unique measure on $(X \times Y, \mathcal{B} \otimes \mathcal{C})$ whose projections on $X$ and $Y$ are equal to $\mu$ and $\nu$ respectively: indeed, we put

$$
\varrho(A \times B)=\int_{B} \Phi\left(\chi_{A}\right) d \nu
$$

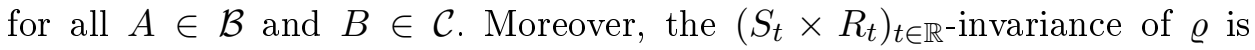
equivalent to

$$
\Phi \circ U_{S_{t}}=U_{R_{t}} \circ \Phi
$$

for each $t \in \mathbb{R}$. Thus we can identify the set of all Markov operators satisfying (4) (denoted by $\mathcal{J}(\mathcal{S}, \mathcal{R})$ ) with the set $J(\mathcal{S}, \mathcal{R})$. The set of Markov operators corresponding to ergodic joinings will be denoted by $\mathcal{J}^{\mathrm{e}}(\mathcal{S}, \mathcal{R})$ (such Markov operators are indecomposable, that is, they are extremal elements of the simplex $\mathcal{J}(\mathcal{S}, \mathcal{R}))$. The notation $\mathcal{J}(\mathcal{S})$ and $\mathcal{J}^{\mathrm{e}}(\mathcal{S})$ is used for self-joinings. Note that $U_{S_{t}} \in \mathcal{J}^{\mathrm{e}}(\mathcal{S}), t \in \mathbb{R}$. Denote by $\int$ the Markov operator corresponding to the product measure, i.e. $\int(f)=\int_{X} f d \mu$.

Following [12], $\mathcal{S}$ and $\mathcal{R}$ are called disjoint (in the sense of Furstenberg) if $J(\mathcal{S}, \mathcal{R})=\{\mu \otimes \nu\}$.

Recall also (see [13]) that whenever the maximal spectral types $\sigma_{\mathcal{S}}$ and $\sigma_{\mathcal{R}}$ of the corresponding Koopman representations on $L_{0}^{2}(X, \mathcal{B}, \mu)$ and $L_{0}^{2}(Y, \mathcal{C}, \nu)$ are mutually singular then $\mathcal{S}$ and $\mathcal{R}$ are disjoint.

Finally, following [9], we say that an ergodic flow $\mathcal{S}=\left(S_{t}\right)_{t \in \mathbb{R}}$ has the ELF-property (briefly, is an ELF-flow) if $\overline{\left\{U_{S_{t}}: t \in \mathbb{R}\right\}} \subset \mathcal{J}^{\mathrm{e}}(\mathcal{S})$ (the closure in the weak operator topology).

1.3. Special flows and ELF-property. Denote by $\mathcal{P}(\mathbb{R})$ the space of all Borel probability measures on $\mathbb{R}$. Recall that a sequence $\left(\sigma_{n}\right)$ of measures 
converges weakly in $\mathcal{P}(\mathbb{R})$ to a measure $\sigma$ if for each function $h \in C B(\mathbb{R})$,

$$
\int_{\mathbb{R}} h d \sigma_{n} \rightarrow \int_{\mathbb{R}} h d \sigma
$$

Let $(X, \mathcal{B}, \mu)$ be a standard probability Borel space. Given a measurable function $f: X \rightarrow \mathbb{R}$, we denote by $f_{*} \mu$ the image of $\mu$ via $f$, i.e. $f_{*} \mu(A)=$ $\mu\left(f^{-1}(A)\right)$ for an arbitrary Borel set $A$ in $\mathbb{R}$.

Note that whenever $\left(f_{k}\right),\left(g_{k}\right)$ are sequences of real measurable functions on $X$ satisfying $\left(f_{k}\right)_{*} \mu \rightarrow \sigma$ as $k \rightarrow \infty$ for some $\sigma \in \mathcal{P}(\mathbb{R})$ and $\mu\{x \in X$ : $\left.f_{k}(x) \neq g_{k}(x)\right\} \rightarrow 0$ as $k \rightarrow \infty$, then $\left(g_{k}\right)_{*} \mu \rightarrow \sigma$ as $k \rightarrow \infty$.

Now we recall basic facts concerning special flows.

Let $T$ be an ergodic automorphism of $(X, \mathcal{B}, \mu)$. We will denote by $\lambda$ Lebesgue measure on $\mathbb{R}$. Assume $f: X \rightarrow \mathbb{R}$ to be a measurable positive function with $\int_{X} f d \mu<\infty$.

We define an $\mathbb{R}$-action, denoted by $T^{f}$, on the space $\left(X^{f}, \mathcal{B}^{f}, \mu^{f}\right)$ where

$$
X^{f}=\{(x, t) \in X \times \mathbb{R}: 0 \leq t<f(x)\},
$$

$\mathcal{B}^{f}$ is the restriction of $\mathcal{B} \otimes \mathcal{B}(\mathbb{R})$ and $\mu^{f}$ is the restriction to $X^{f}$ of the product measure $\mu \otimes \lambda$ of $X \times \mathbb{R}$. This action, denoted by $T^{f}=\left(\left(T^{f}\right)_{t}\right)_{t \in \mathbb{R}}$, will be called the special flow built from $T$ and the roof function $f$ and it is as follows. Each point from $X^{f}$ moves vertically upwards with unit speed and as soon as it reaches the graph of $f$, the point $(x, f(x))$ is identified with $(T x, 0)$ (see e.g. [2, Chapter 11]). Put

$$
f^{(k)}(x)= \begin{cases}f(x)+f(T x)+\cdots+f\left(T^{k-1} x\right) & \text { for } k>0, \\ 0 & \text { for } k=0, \\ -\left(f\left(T^{k} x\right)+\cdots+f\left(T^{-1} x\right)\right) & \text { for } k<0 .\end{cases}
$$

Only functions $f$ satisfying $f \geq c>0$ will be considered. It follows that for a.e. $x \in X$,

$$
f^{(k)}(x) \geq c k, \quad k \in \mathbb{N} .
$$

Recall that a sequence $\left(q_{n}\right)$ is said to be a rigidity time for the automorphism $T$ if $U_{T}^{q_{n}} \rightarrow$ Id as $n \rightarrow \infty$ in the weak (or, equivalently, strong) operator topology.

We now recall some results from [9] that will be needed in what follows.

Proposition 1 ([9]). Suppose that $\mathcal{T}=\left(T_{t}\right)_{t \in \mathbb{R}}$ is an ergodic flow on $(Y, \mathcal{C}, \nu)$ such that for a sequence $\left(t_{n}\right) \subset \mathbb{R}$ with $t_{n} \rightarrow \infty$,

$$
T_{t_{n}} \rightarrow \int_{\mathbb{R}} T_{s} d P(s)
$$

where $P \in \mathcal{P}(\mathbb{R})$. Then

(i) $\mathcal{T}$ is disjoint from all mixing flows, 
(ii) $\mathcal{T}$ is disjoint from all weakly mixing ELF-flows whenever $P$ is not a Dirac measure.

Proposition $2([9])$. Let $\left(q_{n}\right)$ be a rigidity sequence for $T$. Suppose that $f \in L^{2}(X, \mu), f \geq c>0$ and $\int_{X} f(x) d \mu(x)=d<\infty$. Put $f_{0}=$ $f-d$. Moreover, suppose that the sequence $\left(f_{0}^{\left(q_{n}\right)}\right)$ is bounded in $L^{2}(X, \mu)$ and $\left(f_{0}^{\left(q_{n}\right)}\right)_{*} \mu \rightarrow P$ weakly in $\mathcal{P}(\mathbb{R})$. Then

$$
\left(T^{f}\right)_{d q_{n}} \rightarrow \int_{\mathbb{R}}\left(T^{f}\right)_{-t} d P(t)
$$

From the above propositions we obtain the following.

Corollary 1 ([9]). Under the assumptions of Proposition 2 suppose additionally that $T^{f}$ is weakly mixing and that the limit measure $P$ is not a Dirac measure. Then the special flow $T^{f}$ is disjoint from all ELF-flows.

Recall also that if $\left(q_{n}\right)$ is a rigidity time for $T$ and $\left(f_{0}^{\left(q_{n}\right)}\right)_{*} \mu \rightarrow P$ with $P$ continuous then the special flow $T^{f}$ is weakly mixing (see [21]).

We will need some disjointness results concerning flows having integral Markov operators in the weak closure of their time $t$ automorphisms, $t \in \mathbb{R}$.

Lemma 2. Let $\mathcal{T}=\left(T_{t}\right)_{t \in \mathbb{R}}$ and $\mathcal{S}=\left(S_{t}\right)_{t \in \mathbb{R}}$ be $\mathbb{R}$-actions on $(X, \mathcal{B}, \mu)$ and $(Y, \mathcal{C}, \nu)$ respectively. Assume additionally that $\mathcal{T}$ is weakly mixing and $\mathcal{S}$ is ergodic. Moreover, suppose that for a sequence $\left(t_{n}\right) \subset \mathbb{R}$ with $t_{n} \rightarrow \infty$,

$$
T_{t_{n}} \rightarrow \int_{\mathbb{R}} T_{t} d P(t) \quad \text { and } \quad S_{t_{n}} \rightarrow \int_{\mathbb{R}} S_{t} d Q(t) .
$$

If $P \neq Q$ then the flows $\mathcal{T}$ and $\mathcal{S}$ are disjoint in the sense of Furstenberg.

Proof. Let $J: L^{2}(X, \mathcal{B}, \mu) \rightarrow L^{2}(Y, \mathcal{C}, \nu)$ be the Markov operator corresponding to an ergodic joining between $\mathcal{T}$ and $\mathcal{S}$. Thus

$$
J \circ T_{t}=S_{t} \circ J \quad \text { for all } t \in \mathbb{R} .
$$

By taking $t=t_{n}$ and passing to the limits we obtain

$$
J \circ \int_{\mathbb{R}} T_{t} d P(t)=\left(\int_{\mathbb{R}} S_{t} d Q(t)\right) \circ J .
$$

Hence from (6) we have

$$
\int_{\mathbb{R}} J \circ T_{t} d P(t)=\int_{\mathbb{R}} J \circ T_{t} d Q(t) .
$$

Suppose that $J \neq \int$. Denote by $P^{\prime}$ and $Q^{\prime}$ the images via the (continuous) mapping

$$
t \mapsto J \circ T_{t}
$$


of the measures $P$ and $Q$ respectively. We obtain

$$
\begin{aligned}
& \int_{\mathbb{R}} J \circ T_{t} d P(t)=\int_{\mathcal{J}^{\mathrm{e}}(\mathcal{S}, \mathcal{T})} \Phi d P^{\prime}(\Phi), \\
& \int_{\mathbb{R}} J \circ T_{t} d Q(t)=\int_{\mathcal{J}^{\mathrm{e}}(\mathcal{S}, \mathcal{T})} \Phi d Q^{\prime}(\Phi) .
\end{aligned}
$$

In view of (7) and from the uniqueness of the ergodic decomposition we have $P^{\prime}=Q^{\prime}$. Now using weak mixing of $\mathcal{T}$, we conclude that the continuous map (8) is an injection, so (by the Suslin theorem) $P=Q$. The result follows.

Lemma 3. Under the assumptions of Lemma 2 , if $P \neq \delta_{0}$ and $Q=\delta_{0}$, then the flows $\mathcal{T}$ and $\mathcal{S}$ are spectrally disjoint.

Proof. Assume that the (reduced) maximal spectral types $\sigma_{U_{\mathcal{T}}}$ and $\sigma_{U_{\mathcal{S}}}$ of $\mathcal{T}$ and $\mathcal{S}$ are not mutually singular. Then there exists a probability measure $\sigma$ such that $\sigma \ll \sigma_{U_{\mathcal{T}}}$ and $\sigma \ll \sigma_{U_{\mathcal{S}}}$. We have $\sigma=\sigma_{f, U_{\mathcal{T}}}=\sigma_{g, U_{\mathcal{S}}}$ for some functions $f \in L_{0}^{2}(X, \mathcal{B}, \mu)$ and $g \in L_{0}^{2}(Y, \mathcal{C}, \nu)$ and $\|f\|=\|g\|=1$. From the assumptions we obtain

$$
\begin{aligned}
& \widehat{\sigma}\left(t_{n}\right)=\left\langle T_{t_{n}} f, f\right\rangle \rightarrow \int_{\mathbb{R}}\left\langle T_{t} f, f\right\rangle d P(t), \\
& \widehat{\sigma}\left(t_{n}\right)=\left\langle S_{t_{n}} g, g\right\rangle \rightarrow \int_{\mathbb{R}}\left\langle S_{t} g, g\right\rangle d \delta_{0}(t)=\|g\|^{2}=1 .
\end{aligned}
$$

A convexity argument shows immediately that $\left\langle T_{t} f, f\right\rangle=1$ for $P$-a.e. $t \in \mathbb{R}$. Now the equality in the Schwarz inequality and the weak mixing of $\mathcal{T}$ yield $f=0$.

REMARK 1. Note that the proof of Corollary 5.2 from [9] gives rise to the following: If for flows $\mathcal{T}=\left(T_{t}\right)_{t \in \mathbb{R}}, \mathcal{S}=\left(S_{t}\right)_{t \in \mathbb{R}}$, one of which is weakly mixing, we have

$$
T_{t_{n}} \rightarrow \int T_{t} d P(t), \quad S_{t_{n}} \rightarrow \int S_{t} d Q(t)
$$

for some $t_{n} \rightarrow \infty, P \neq Q$ and $P$ and $Q$ have compact supports then $\mathcal{T}$ and $\mathcal{S}$ are spectrally disjoint.

Indeed, otherwise, as in the proof of the above lemma, we obtain

$$
\widehat{\sigma}\left(t_{n}\right) \rightarrow \int_{\mathbb{R}}\left\langle T_{t} f, f\right\rangle d P(t)=\int_{\mathbb{R}}\left\langle S_{t} g, g\right\rangle d Q(t) .
$$

But

$$
\int_{\mathbb{R}}\left\langle T_{t} f, f\right\rangle d P(t)=\int_{\mathbb{R}} \widehat{P}(s) d \sigma_{f, U_{\mathcal{T}}}(s)=\int_{\mathbb{R}} \widehat{P}(s) d \sigma(s)
$$

and hence $\int_{\mathbb{R}} \widehat{P}(s) d \sigma(s)=\int_{\mathbb{R}} \widehat{Q}(s) d \sigma(s)$. Moreover, we can repeat the same reasoning for each probability measure $\eta \ll \sigma$ and therefore $\widehat{P}(s)=\widehat{Q}(s)$ for $\sigma$-a.e. $s \in \mathbb{R}$. Since $P$ and $Q$ have compact supports, $\widehat{P}$ and $\widehat{Q}$ have 
analytic extensions to the whole complex plane. Since $\sigma$ is continuous, $\widehat{P}=\widehat{Q}$ everywhere, and hence $P=Q$.

Finally, notice that the set of integral Markov operators is closed under the weak convergence of measures: if $P_{n}$ converges weakly to $P$ in $\mathcal{P}(\mathbb{R})$ then

$$
\int_{\mathbb{R}} T_{t} d P_{n}(t) \rightarrow \int_{\mathbb{R}} T_{t} d P(t)
$$

Indeed, we only need to check that for each $f, g \in L^{2}(X, \mathcal{B}, \mu)$,

$$
\begin{aligned}
\left\langle\left(\int_{\mathbb{R}} T_{t} d P_{n}(t)\right) f, g\right\rangle=\int_{\mathbb{R}}\left\langle T_{t} f, g\right\rangle d P_{n}(t) & \\
& \rightarrow \int_{\mathbb{R}}\left\langle T_{t} f, g\right\rangle d P(t)=\left\langle\left(\int_{\mathbb{R}} T_{t} d P(t)\right) f, g\right\rangle,
\end{aligned}
$$

which is immediate as the function $\mathbb{R} \ni t \mapsto\left\langle T_{t} f, g\right\rangle$ is continuous and bounded.

\section{Analytic special flows disjoint from the ELF-class}

2.1. Generic method. Let $f:[0,1) \rightarrow \mathbb{R}$ be a real-analytic function. Consider its Fourier expansion

$$
f(x)=\sum_{n=-\infty}^{\infty} b_{n} e^{2 \pi i n x}
$$

where $b_{n}=\mathrm{o}\left(A^{|n|}\right)$ for some $0<A<1, n \in \mathbb{Z}$. We additionally require that $\int f=0\left(b_{0}=0\right)$ and that $\#\left\{n \in \mathbb{Z}: b_{n} \neq 0\right\}=\infty$ (so that $f$ is not a trigonometric polynomial).

Lemma 4. There exists an infinite sequence $\left(q_{n}\right)$ such that $b_{q_{n}} \neq 0$ and

$$
\lim _{n \rightarrow \infty} \frac{\left|b_{q_{n}}\right|}{\left|b_{q_{n}}\right|+\left|b_{2 q_{n}}\right|+\cdots}=1
$$

Proof. Choose $q_{1} \geq 1$ and $\delta_{1}>0$ so that

$$
\frac{\left|b_{q_{1}}\right|}{A^{q_{1}}} \geq \delta_{1} \quad \text { and } \quad \frac{\left|b_{s}\right|}{A^{s}}<\delta_{1} \quad \text { for all } s>q_{1} .
$$

Then

$$
\sum_{i=2}^{\infty}\left|b_{i q_{1}}\right|<\sum_{i=2}^{\infty} A^{i q_{1}} \delta_{1}=\delta_{1} A^{q_{1}} \frac{A^{q_{1}}}{1-A^{q_{1}}} \leq\left|b_{q_{1}}\right| \frac{A^{q_{1}}}{1-A^{q_{1}}} .
$$

Now choose $q_{2}>q_{1}$ and $\delta_{2}>0$ so that (10) holds with $q_{2}$ instead of $q_{1}$ and $\delta_{2}$ instead of $\delta_{1}$. It follows that we can choose an increasing sequence $\left(q_{n}\right)$ for which

$$
\sum_{i=2}^{\infty}\left|b_{i q_{n}}\right| \leq\left|b_{q_{n}}\right| \frac{A^{q_{n}}}{1-A^{q_{n}}}=\mathrm{o}\left(\left|b_{q_{n}}\right|\right),
$$

and the proof is complete. 
For a fixed integer $q \geq 1$ consider now a $1 / q$-periodic function $f_{q}:[0,1) \rightarrow$ $\mathbb{R}$ given by

$$
f_{q}(x)=f(x)+f\left(x+\frac{1}{q}\right)+\cdots+f\left(x+\frac{q-1}{q}\right) .
$$

By the Koksma inequality, $f_{q} \rightarrow 0$ uniformly as $q \rightarrow \infty$, in particular

$$
\left(f_{q}\right)_{*} \mu \rightarrow \delta_{0} \quad \text { as } q \rightarrow \infty .
$$

Moreover,

$$
f_{q}(x)=q \sum_{l=-\infty}^{\infty} b_{l q} e^{2 \pi i l q x} .
$$

We will consider functions of the form $s f_{q}$ for some positive integer $s$. We have

$$
s f_{q}(x)=s q 2 \operatorname{Re}\left(b_{q} e^{2 \pi i q x}\right)+s q 2 \operatorname{Re}\left(b_{2 q} e^{2 \pi i 2 q x}+b_{3 q} e^{2 \pi i 3 q x}+\cdots\right) .
$$

Since $b_{q} \rightarrow 0$ exponentially fast, $q b_{q} \rightarrow 0$ as $q \rightarrow \infty$. Hence $q_{n} b_{q_{n}} \rightarrow 0$, where $\left(q_{n}\right)$ is the sequence from Lemma 4 . We now select a sequence $\left(s_{n}\right)$ of natural numbers so that

$$
s_{n} q_{n}\left|b_{q_{n}}\right| \rightarrow 1 \quad \text { as } n \rightarrow \infty .
$$

It follows from (11) and Lemma 4 that

$$
s_{n} q_{n}\left(\left|b_{2 q_{n}}\right|+\left|b_{3 q_{n}}\right|+\cdots\right) \rightarrow 0 \quad \text { as } n \rightarrow \infty .
$$

Proposition 3. The sequence of distributions $\left(s_{n} f_{q_{n}}\right)_{*} \mu$ converges weakly to $\sigma$, where $\sigma$ is an absolutely continuous measure on $[-2,2]$. Furthermore

$$
\left\|s_{n} f_{q_{n}}\right\|_{C([0,1))} \leq 3 \quad \text { for } n \geq n_{0} .
$$

Proof. Since $\left|s_{n} q_{n} b_{q_{n}}\right| \rightarrow 1$ as $n \rightarrow \infty$, the sequence of distributions $\left(s_{n} q_{n} b_{q_{n}} e^{2 \pi i q_{n} x}\right)_{*} \mu$ tends to Lebesgue measure on $\mathbb{T}$. It follows that $\left(2 \operatorname{Re}\left(s_{n} q_{n} b_{q_{n}} e^{2 \pi i q_{n} x}\right)\right)_{*} \mu \rightarrow \widetilde{\sigma}$, where $\widetilde{\sigma}$ is the image of Lebesgue measure from $\mathbb{T}$ via the map $z \mapsto 2 \operatorname{Re} z$. In view of (12),

$$
2 \operatorname{Re}\left(s_{n} q_{n} b_{2 q_{n}} e^{2 \pi i 2 q_{n} x}+s_{n} q_{n} b_{3 q_{n}} e^{2 \pi i 3 q_{n} x}+\cdots\right) \rightarrow 0 \quad \text { as } n \rightarrow \infty
$$

uniformly, so the corresponding sequence of distributions converges to the Dirac measure $\delta_{0}$. In particular $s_{n} f_{q_{n}}$ and $2 \operatorname{Re}\left(s_{n} q_{n} b_{q_{n}} e^{2 \pi i q_{n} x}\right)$ have the same limit distributions as their difference tends to zero in measure. Therefore $\left(s_{n} f_{q_{n}}\right)_{*} \mu \rightarrow \widetilde{\sigma}$. The uniform bound of $s_{n} f_{q_{n}}$ is obvious from (13) and (11).

In view of Lemma 1 the set of $\alpha$ 's for which $\left|\alpha-p_{n} / q_{n}\right|<r\left(q_{n}\right)$ for infinitely many $n$ 's, where $r\left(q_{n}\right)=\mathrm{o}\left(\left|b_{q_{n}}\right|^{2}\right)$ and $p_{n} / q_{n}$ are the convergents of $\alpha$, is residual. For an irrational $\alpha$ in this set, $\left(s_{n} q_{n}\right)$ is a rigidity time for $T x=x+\alpha$. Moreover, for some infinite subsequence $\left(n_{k}\right)$ we get

$$
\left|s_{n_{k}} f_{q_{n_{k}}}(x)-f^{\left(s_{n_{k}} q_{n_{k}}\right)}(x)\right| \rightarrow 0 \quad \text { as } k \rightarrow \infty
$$


uniformly in $x \in[0,1)$. Indeed (to simplify notation we write $q$ and $s$ instead of $q_{n_{k}}$ and $s_{n_{k}}$ respectively),

$$
\begin{aligned}
s f_{q}(x)-f^{(s q)}(x)= & \sum_{j=1}^{s}\left(\left[f(x)+f\left(x+\frac{1}{q}\right)+\cdots+f\left(x+\frac{q-1}{q}\right)\right]\right. \\
& -[f(x+(j-1) q \alpha)+f(x+(j-1) q \alpha+\alpha) \\
& +\cdots+f(x+(j-1) q \alpha+(q-1) \alpha)]) .
\end{aligned}
$$

Given $1 \leq j \leq s$ and $0 \leq k \leq q-1$, for some $i \in\{(j-1) q, \ldots, j q-1\}$ we have $k / q=i p / q(\bmod 1)$ and then

$$
\left|f\left(x+\frac{k}{q}\right)-f(x+i \alpha)\right| \leq\left\|f^{\prime}\right\|_{C([0,1))} i\left|\frac{p}{q}-\alpha\right| \leq\left\|f^{\prime}\right\|_{C([0,1))} \operatorname{sqr}(q) .
$$

Now in view of (11),

$$
\left|s f_{q}(x)-f^{(s q)}(x)\right| \leq s q\left\|f^{\prime}\right\|_{C([0,1))} s q r(q)=\left\|f^{\prime}\right\|_{C([0,1))}\left(s q\left|b_{q}\right|\right)^{2} \frac{r(q)}{\left|b_{q}\right|^{2}} \rightarrow 0
$$

as $q \rightarrow \infty$, which completes the proof of (14).

Thus we have proved the following.

Proposition 4. There exists a residual subset of $[0,1)$ such that whenever an irrational $\alpha$ belongs to this set there exists a sequence $\left(q_{n}\right)$ (a subsequence of denominators of $\alpha$ ) such that for some sequence $\left(s_{n}\right),\left(s_{n} q_{n}\right)$ is a rigidity time for $T$, the sequence of distributions $\left(f^{\left(s_{n} q_{n}\right)}\right)_{*} \mu$ tends to an absolutely continuous measure on $[-2,2]$ and the sequence of norms $\left(\left\|f^{\left(s_{n} q_{n}\right)}\right\|_{C([0,1))}\right)$ is bounded.

Take now the function $\tilde{f}=f+d$ for some $d$ so that $\tilde{f}>0$. Then $\widetilde{f}_{0}=f$ and $\widetilde{f}$ (with the sequence $\left.\left(s_{n} q_{n}\right)_{n}\right)$ satisfies the assumptions of Corollary 1 .

2.2. The a.a.c.c.p. ("almost analytic cocycle construction procedure"). First, we briefly recall the a.a.c.c.p. from [17], with a small modification for the purpose of this paper (see Remark 2 below).

The aim of an a.a.c.c.p. is to construct a (non-trivial) cocycle of the form $\varphi=\sum_{k=1}^{\infty} \varphi_{k}$, where $\varphi_{k}$ 's are step-cocycles which are coboundaries and $\varphi$ is cohomologous to some analytic cocycle $f$.

We are given a collection of the following parameters: a sequence $\left(M_{k}\right)$ $\subset \mathbb{N}$ and an array $\left(\left(d_{k 1}, \ldots, d_{k M_{k}}\right)\right)_{k \geq 1}$ with $d_{k i} \in \mathbb{R}$ such that for each $k$,

$$
\sum_{i=1}^{M_{k}} d_{k i}=0
$$


and

$$
M_{k} \geq 2\left(\sum_{i=1}^{k-1} D_{i} M_{i}\right)^{2}
$$

where $D_{k}=\max _{1 \leq i \leq M_{k}}\left|d_{k i}\right|$. Then we choose a sequence $\left(\varepsilon_{k}\right) \subset \mathbb{R}_{+}$such that $\sum_{k=1}^{\infty} \sqrt{\varepsilon_{k}} M_{k}<\infty, \sum_{k=1}^{\infty} \varepsilon_{k}<1$ and $\varepsilon_{k}<1 / D_{k}^{2}$ for $k=1,2, \ldots$ The last needed parameter is a real number $A>1$.

Having collected the above parameters, we say that the a.a.c.c.p. is realized over an irrational number $\alpha=\left[0 ; a_{1}, a_{2}, \ldots\right]$ if there exists a strictly increasing sequence $\left(n_{k}\right) \subset \mathbb{N}$ satisfying

$$
A^{N_{k}} \frac{D_{k} M_{k}}{a_{2 n_{k}+1} q_{2 n_{k}}}<\frac{1}{2^{k}}
$$

where $N_{k}$ is the degree of a real trigonometric polynomial

$$
P_{k}(t)=\sum_{s=-N_{k}}^{N_{k}} b_{s}^{(k)} e^{2 \pi i s t}
$$

such that $\int_{0}^{1} P_{k}(t) d t=1, P_{k} \geq 0$, and $P_{k}(t)<\varepsilon_{k}$ for $t \in\left(\eta_{k} / 2,1\right)$ (notice that $\left.\left|b_{s}^{(k)}\right| \leq\left\|P_{k}\right\|_{L^{1}}=1\right)$, where the $\eta_{k}$ 's satisfy $4 M_{k} \eta_{k}<\varepsilon_{k} / q_{2 n_{k}}$. Furthermore, we require that $a_{2 n_{k}+1}>2$ and $1 / a_{2 n_{k}+1} q_{2 n_{k}}<\frac{1}{2} \eta_{k}$.

Now we can define a cocycle $\varphi=\sum_{k=1}^{\infty} \varphi_{k}$ in the following way. In the interval $I_{k}=\left[0,\left\{a_{2 n_{k}+1} q_{2 n_{k}} \alpha\right\}\right)$ we will choose pairwise disjoint intervals $\omega_{k 1}, \ldots, \omega_{k M_{k}}$ of the same length $\lambda_{k} \in\left(\eta_{k}, 2 \eta_{k}\right)$ such that each $\omega_{k i}$ consists of $e_{k} \geq 3$ (odd) consecutive subintervals $J_{t}^{k}$. For $i=1, \ldots, M_{k}-1$ the interval $\omega_{k, i+1}$ follows $\omega_{k i}$ in the natural ordering of $[0,1)$ and, in general, they are separated by a certain number of consecutive subintervals $J_{j}^{k}$. Denoting by $J_{s_{k, i}}^{k}$ the central subinterval in $\omega_{k i}$ define

$$
\varphi_{k}(x)= \begin{cases}d_{k i} & \text { for } x \in J_{s_{k, i}}^{k}, i=1, \ldots, M_{k}, \\ 0 & \text { otherwise. }\end{cases}
$$

Since $I_{k+1} \subset J_{1}^{k}$, the $\varphi_{k}$ 's have disjoint supports and $\varphi=\sum_{k=1}^{\infty} \varphi_{k}$ is well defined.

REMARK 2. In comparison with [17] we slightly reduced the number of conditions imposed on a.a.c.c.p. (for example (8) in [17] follows from (7) and (10) there, $\left\|P_{k}\right\|_{\mathcal{F}}=1$ ), however we added a new condition (16). In what follows we will still add some extra conditions on $M_{k}$ and $d_{k i}$. It is however clear that the argument contained in the proof of Proposition 1 in [17] persists, and the set of $\alpha$ 's over which an a.a.c.c.p. (in the sense of the present paper) is realized is a $G_{\delta}$ and dense subset of $[0,1)$. Recall that the argument used in [17] was a simple observation that the a.a.c.c.p. can be 
carried out over an $\alpha=\left[0 ; a_{1}, a_{2}, \ldots\right]$ whenever for a subsequence $\left(n_{k}\right)$ the $a_{2 n_{k}+1}$ 's are sufficiently large. This also says that we can find an $\alpha$ for which we can require the $a_{2 n_{k}+1}$ 's to have a special form (for example be multiples of $M_{k}$, etc.)

REMARK 3. Assume that an a.a.c.c.p. with an array $\left(d_{k i}\right)$ is realized over an irrational $\alpha$. We now take another a.a.c.c.p. which has the same sequences of parameters except for an array $\left(d_{k i}\right)$ which is now $\left(d_{k i}^{\prime}\right)$. Then the second a.a.c.c.p. is realized over the same $\alpha$ provided that $D_{k}^{\prime} \leq D_{k}$, $k \geq 1$. In other words, to define $\alpha$ we need the sequence $\left(D_{k}\right)$ rather than the sequence $\left(\left(d_{k 1}, \ldots, d_{k M_{k}}\right)\right)$.

Moreover, the following theorem holds true.

THEOREM 1 ([17]). Suppose that for an irrational $\alpha$ an a.a.c.c.p. is realized. Then there exists an analytic cocycle $f:[0,1) \rightarrow \mathbb{R}$ which is $\alpha$ cohomologous to $\varphi$.

REMARK 4. In the proof of Theorem 1 in [17] an explicit form of the analytic cocycle $f$ (cohomologous to $\varphi$ ) has been given. Namely

$$
f(t)=\sum_{k=1}^{\infty} l_{k} \sum_{r=1}^{M_{k}} d_{k r} P_{k}\left(t-m_{k r} \alpha\right),
$$

where $m_{k r}$ is determined by $J_{s_{k, r}}^{k}=T^{m_{k r}} J_{1}^{k}$. Recall that $l_{k}=\left|J_{1}^{k}\right|$. Thus the Fourier expansion of $f$ is given by

$$
f(t)=\sum_{s=-\infty}^{\infty}\left(\sum_{\left\{k:|s| \leq N_{k}\right\}} b_{s}^{(k)} l_{k} \sum_{r=1}^{M_{k}} d_{k r} e^{-2 \pi i s m_{k r} \alpha}\right) e^{2 \pi i s t} .
$$

This allows us to show (see the lemma below) that the analytic functions $f$ obtained from the a.a.c.c.p. enjoy a universal bound which depends only on $A>1$.

Lemma 5. For each $f$ given by $(18),|f(t)| \leq 2 /(A-1)$ for each $t \in[0,1)$.

Proof. From (19), (2) and (17), for $s \neq 0$ we have

$$
\begin{aligned}
\left|\widehat{f}_{s}\right| & =\left|\sum_{\left\{k:|s| \leq N_{k}\right\}} l_{k} b_{s}^{(k)} \sum_{r=1}^{M_{k}} d_{k r} e^{-2 \pi i s m_{k r} \alpha}\right| \\
& \leq \sum_{\left\{k:|s| \leq N_{k}\right\}} l_{k} M_{k} D_{k} \frac{A^{N_{k}}}{A^{N_{k}}} \leq \frac{1}{A^{|s|}} \sum_{k=1}^{\infty} \frac{1}{2^{k}}=\frac{1}{A^{|s|}} .
\end{aligned}
$$

Thus

$$
|f(t)| \leq \sum_{s=-\infty}^{\infty}\left|\widehat{f}_{s}\right| \leq 2 \sum_{s=1}^{\infty} \frac{1}{A^{s}}=\frac{2}{A-1} .
$$


We will also need the following property of the a.a.c.c.p.

Lemma 6 ([17]). For an arbitrary a.a.c.c.p., an arbitrary $\alpha$ over which it is realized and for any $k$, the cocycle $\varphi$ is constant on each interval $T^{i}\left(I_{k}\right)$, $i=1, \ldots, q_{2 n_{k}}-1$. Moreover, if we put $b_{k, i}=\varphi \mid T^{i}\left(I_{k}\right)$ then

$$
\sum_{i=1}^{q_{2 n_{k}}-1} b_{k, i}=0
$$

Now, we will carry out an a.a.c.c.p., selecting the parameters so that the sequence of distributions $\left(\varphi^{\left(b_{k} q_{2 n_{k}}\right)}\right)_{*} \mu$ tends to a continuous measure on $[-1,1]$, where $\left(b_{k} q_{2 n_{k}}\right)$ is a certain rigidity time for $T$.

Fix an arbitrary probability continuous measure $\nu$ on $[-1,1]$. We will put some extra conditions on $M_{k}, d_{k i}$ :

$$
\begin{gathered}
\left|d_{k i}\right| \leq 1, \quad i=1, \ldots, M_{k}, k \geq 1 ; \\
M_{k}^{-1} \sum_{i=1}^{M_{k}} \delta_{d_{k i}} \rightarrow \nu \quad \text { weakly in } \mathcal{P}([-1,1]) \text { as } k \rightarrow \infty,
\end{gathered}
$$

in particular (from (21))

$$
M_{k} \rightarrow \infty \quad \text { as } k \rightarrow \infty .
$$

Let $\alpha$ be an irrational over which the a.a.c.c.p. is carried out. We require additionally that $a_{2 n_{k}+1}$ is a multiple of $M_{k}$, more precisely

$$
a_{2 n_{k}+1}=M_{k} b_{k}
$$

where $b_{k}$ is odd, $k \geq 1$.

We now define $\varphi_{k}$ 's by putting

$$
s_{k, i}=(i-1) b_{k}+\frac{b_{k}+1}{2}, \quad i=1, \ldots, M_{k},
$$

and then defining

$$
\varphi_{k}(x)=d_{k i} \quad \text { if } x \in J_{s_{k, i}}^{k}, i=1, \ldots, M_{k},
$$

and 0 elsewhere (notice that $b_{k} l_{k}$ is of order

$$
\frac{a_{2 n_{k}+1}}{M_{k}} \cdot \frac{1}{a_{2 n_{k}+1} q_{2 n_{k}}}=\frac{1}{M_{k} q_{2 n_{k}}}
$$

while $\eta_{k}$ is at most of order $\varepsilon_{k} / M_{k} q_{2 n_{k}}$, so the intervals $\omega_{k 1}, \ldots, \omega_{k M_{k}}$ are disjoint).

Set $\xi_{j}=J_{(j-1) b_{k}+1}^{k} \cup \cdots \cup J_{j b_{k}}^{k}, j=1, \ldots, M_{k}$ (that is, $\xi_{1}, \ldots, \xi_{M_{k}}$ are consecutive intervals of equal length $b_{k} l_{k}$ partitioning $I_{k}$ ).

Consider now the union

$$
\bigcup_{s=1}^{q_{2 n_{k}}} T^{s-q_{2 n_{k}}\left(b_{k}+1\right) / 2} \xi_{j}=: U_{k, j}, \quad j=1, \ldots, M_{k} .
$$


We obtain

$$
\varphi_{k}^{\left(b_{k} q_{2 n_{k}}\right)}(x)=d_{k j}, \quad x \in U_{k, j}, j=1, \ldots, M_{k} .
$$

Recall that $\Sigma_{k}=[0,1) \backslash \bigcup_{s=0}^{q_{2 n_{k}}-1} T^{s} I_{k}$ and from (3) and (22),

$$
\mu\left(\Sigma_{k}\right) \rightarrow 0 \quad \text { as } k \rightarrow \infty \text {. }
$$

Observe that $\varphi_{k}^{\left(b_{k} q_{2 n_{k}}\right)}=0$ on the complement of the set $\bigcup_{j=1}^{M_{k}} U_{k, j}$ which has the same measure as $\Sigma_{k}$.

Consider now the sequence of distributions $\left(\varphi_{k}^{\left(b_{k} q_{2 n_{k}}\right)}\right)_{*} \mu$. We will show that $\left(\varphi_{k}^{\left(b_{k} q_{2 n_{k}}\right)}\right)_{*} \mu \rightarrow \nu$ as $k \rightarrow \infty$. Indeed, observe that

$$
\left(\varphi_{k}^{\left(b_{k} q_{2 n_{k}}\right)}\right)_{*} \mu=\sum_{j=1}^{M_{k}} \mu\left(U_{k, j}\right) \delta_{d_{k j}}+\mu\left(\Sigma_{k}\right) \delta_{0}
$$

where $\mu\left(U_{k, j}\right)=\left(1-\mu\left(\Sigma_{k}\right)\right) / M_{k}$. Now using (25) and (21) we get

$$
\left(\varphi_{k}^{\left(b_{k} q_{2 n_{k}}\right)}\right)_{*} \mu=\frac{1-\mu\left(\Sigma_{k}\right)}{M_{k}} \sum_{j=1}^{M_{k}} \delta_{d_{k j}}+\mu\left(\Sigma_{k}\right) \delta_{0} \rightarrow \nu \quad \text { as } k \rightarrow \infty .
$$

So we have constructed a cocycle $\varphi=\sum_{k=1}^{\infty} \varphi_{k}$ for which the sequence of distributions $\left(\varphi_{k}^{\left(b_{k} q_{2 n_{k}}\right)}\right)_{*} \mu$ converges weakly to $\nu$.

We will now show that

$$
\mu\left(\left\{x \in[0,1): \varphi_{k}^{\left(b_{k} q_{2 n_{k}}\right)}(x) \neq \varphi^{\left(b_{k} q_{2 n_{k}}\right)}(x)\right\}\right) \rightarrow 0 \quad \text { as } k \rightarrow \infty .
$$

Indeed, putting $w_{k}=b_{k} q_{2 n_{k}}$ we get

$$
\varphi^{\left(w_{k}\right)}(x)=\sum_{i=1}^{\infty} \varphi_{i}^{\left(w_{k}\right)}(x) .
$$

Notice that the support of $\sum_{i \geq k+1} \varphi_{i}$ is contained in $I_{k+1} \subset J_{1}^{k}$, therefore it follows from (2) and (22) that

$\mu\left(\left\{x \in[0,1): \sum_{i \geq k+1} \varphi_{i}^{\left(w_{k}\right)}(x) \neq 0\right\}\right) \leq l_{k} b_{k} q_{2 n_{k}}<\frac{b_{k}}{a_{2 n_{k}+1}} \rightarrow 0 \quad$ as $k \rightarrow \infty$.

On the other hand, $\sum_{i=1}^{k-1} \varphi_{i}$ is zero on $I_{k}$, which combined with Lemma 6 implies

$$
\mu\left(\left\{x \in[0,1): \sum_{i=1}^{k-1} \varphi_{i}^{\left(w_{k}\right)}(x) \neq 0\right\}\right) \leq \mu\left(\Sigma_{k}\right)+\mu\left(\bigcup_{s=0}^{w_{k}-1} T^{s} J_{a_{2 n_{k}+1}-b_{k}+1}^{k}\right)
$$

hence

$$
\mu\left(\left\{x \in[0,1): \sum_{i=1}^{k-1} \varphi_{i}^{\left(w_{k}\right)}(x) \neq 0\right\}\right) \rightarrow 0 \quad \text { as } k \rightarrow \infty
$$


Thus (26) follows. We hence proved that

$$
\left(\varphi^{\left(b_{k} q_{2 n_{k}}\right)}\right)_{*} \mu \rightarrow \nu \quad \text { as } k \rightarrow \infty \text {. }
$$

Clearly

$$
\left\|b_{k} q_{2 n_{k}} \alpha\right\| \leq \frac{b_{k}}{q_{2 n_{k}+1}} \leq \frac{a_{2 n_{k}+1}}{M_{k}} \cdot \frac{1}{a_{2 n_{k}+1} q_{2 n_{k}}} \rightarrow 0
$$

so $\left(b_{k} q_{2 n_{k}}\right)$ is a rigidity sequence for $T x=x+\alpha$. For all assumptions of Corollary 1 to be satisfied we need to check that $\left(\left\|\varphi^{\left(w_{k}\right)}\right\|_{L^{2}}\right)_{k}$ is bounded.

Notice that since $\left|\varphi_{i}\right| \leq 1$ for each $i \in \mathbb{N}$ and the $\varphi_{i}$ 's have disjoint supports, we have

We have

$$
\left\|\sum_{i \geq k+1} \varphi_{i}\right\|_{\infty} \leq 1
$$

$$
\varphi^{\left(w_{k}\right)}(x)=\left(\sum_{i=1}^{k-1} \varphi_{i}\right)^{\left(w_{k}\right)}(x)+\varphi_{k}^{\left(w_{k}\right)}(x)+\left(\sum_{i \geq k+1} \varphi_{i}\right)^{\left(w_{k}\right)}(x) .
$$

From the construction it follows that

$$
\left|\varphi_{k}^{\left(w_{k}\right)}(x)\right| \leq 1 \quad \text { for all } x \in[0,1) .
$$

Taking into account (28) and the facts that $\operatorname{supp}\left(\sum_{i \geq k+1} \varphi_{i}\right) \subset I_{k+1}$ and that $\operatorname{card}\left(\left\{x, x+\alpha, \ldots, x+\left(w_{k}-1\right) \alpha\right\} \cap I_{k+1}\right) \leq 1$ for all $x \in[0,1)$, we get

$$
\left\|\left(\sum_{i \geq k+1} \varphi_{i}\right)^{\left(w_{k}\right)}\right\|_{\infty} \leq 1
$$

Consider $\left(\sum_{i=1}^{k-1} \varphi_{i}\right)^{\left(w_{k}\right)}$. Notice that because of (15), given $i \geq 1$ and $x \in$ $[0,1)$, we have $\left|\varphi_{i}^{(n)}(x)\right| \leq \sum_{j=1}^{M_{i}}\left|d_{i j}\right| \leq D_{i} M_{i}$ for all $n \geq 0$. Hence in view of $(27),(3)$ and (16), we obtain

$$
\begin{aligned}
\left\|\left(\sum_{i=1}^{k-1} \varphi_{i}\right)^{\left(w_{k}\right)}\right\|_{L^{2}} & \leq\left(\sum_{i=1}^{k-1} D_{i} M_{i}\right) \cdot\left(\mu\left(\left\{x \in[0,1): \sum_{i=1}^{k-1} \varphi_{i}^{\left(w_{k}\right)}(x) \neq 0\right\}\right)\right)^{1 / 2} \\
& \leq\left(\sum_{i=1}^{k-1} D_{i} M_{i}\right) \cdot\left(\mu\left(\Sigma_{k}\right)+\mu\left(\bigcup_{s=0}^{w_{k}-1} T^{s} J_{a_{2 n_{k}+1}-b_{k}+1}^{k}\right)\right)^{1 / 2} \\
& \leq\left(\sum_{i=1}^{k-1} D_{i} M_{i}\right) \cdot\left(\frac{2}{a_{2 n_{k}+1}}+\frac{b_{k}}{a_{2 n_{k}+1}}\right)^{1 / 2} \\
& \leq\left(\sum_{i=1}^{k-1} D_{i} M_{i}\right) \sqrt{\frac{2}{M_{k}}} \leq 1
\end{aligned}
$$

We have obtained a zero mean cocycle $\varphi:[0,1) \rightarrow \mathbb{R}$ with $|\varphi| \leq 1$ satisfying $\left(\varphi^{\left(b_{k} q_{2 n_{k}}\right)}\right)_{*} \mu \rightarrow \nu$, where $\left(b_{k} q_{2 n_{k}}\right)$ is a rigidity time for the rotation $T$ and moreover $\left(\left\|\varphi^{\left(b_{k} q_{2 n_{k}}\right)}\right\|_{L^{2}}\right)$ is bounded. Moreover, from Theorem 1 such a 
cocycle is cohomologous to some real-analytic function $f$. Since $\int \varphi d \mu=0$, we have $\int f d \mu=0$. Let $d>0$ be such that $\widetilde{\varphi}=\varphi+d>0$ and $\widetilde{f}=f+d>0$. Then $\int \widetilde{\varphi} d \mu=d$ and $\int \widetilde{f} d \mu=d$. Note that $\widetilde{\varphi}_{0}=\widetilde{\varphi}-\int \widetilde{\varphi}=\varphi$ and similarly $\tilde{f}_{0}=f$.

Thus we have proved the following.

COROLlary 2. The special flow $T^{\widetilde{\varphi}}$ built from the rotation $T$ and the roof function $\widetilde{\varphi}$ is disjoint from all ELF-flows.

Since $\tilde{f}$ and $\widetilde{\varphi}$ are cohomologous, $T^{\widetilde{f}}$ and $T^{\widetilde{\varphi}}$ are isomorphic. In particular $T^{\widetilde{f}}$ is disjoint from an arbitrary ELF-flow.

3. Analytic flows on the torus which are disjoint in the sense of Furstenberg from all ELF-flows. In Section 2 we have presented two different methods to obtain a special flow $T^{\widetilde{f}}$ with an analytic roof function $\widetilde{f}$ and over an irrational rotation $T$, which is disjoint in the sense of Furstenberg from an arbitrary ELF-flow. We will now show that whenever $d>\pi /(A-1)$ then $T^{\widetilde{f}}(\widetilde{f}=f+d)$ is a natural special representation of some flow $\mathcal{T}=$ $\left(T_{t}\right)_{t \in \mathbb{R}}$ on the torus $[0,1)^{2}$ given by

$$
T_{t}(x, y)=(x(t), y(t))
$$

where $(x(t), y(t))$ is the only solution of the system of differential equations

$$
\left\{\begin{array}{l}
\frac{d x}{d t}=\frac{\alpha}{F(x, y)}, \\
\frac{d y}{d t}=\frac{1}{F(x, y)} .
\end{array}\right.
$$

Here $F:[0,1)^{2} \rightarrow \mathbb{R}_{+}$is analytic and $(x(0), y(0))=(x, y)$ and the function $F$ will be precisely defined by (30) below. Recall first some facts concerning flows arising from (29) (see [2, Chapter 16] for details).

Let us assume that the function $F$ in (29) is smooth. Notice that from the Liouville theorem, the flow $\mathcal{T}$ preserves the measure $F(x, y) d x d y$. Furthermore $\mathcal{T}$ is ergodic since it arises from a change of time of the linear flow which is ergodic. Moreover, the following holds.

Proposition 5 ([2]). The flow $\mathcal{T}=\left(T_{t}\right)_{t \in \mathbb{R}}$ corresponding to the system (29) is isomorphic to the special flow $T^{h}$, where

$$
h(x)=\int_{0}^{1} F(x+s \alpha, s) d s .
$$

Conversely, having a special flow $T^{h}$, where $h$ is smooth, we can find a smooth function $F:[0,1)^{2} \rightarrow \mathbb{R}_{+}$such that $T^{h}$ is isomorphic to $\mathcal{T}=\left(T_{t}\right)_{t \in \mathbb{R}}$ coming from (29). 
Assume now that $h(x)=\sum_{k=-\infty}^{\infty} \widehat{h}_{k} e^{2 \pi i k x}$ is analytic and $\int_{0}^{1} h d \mu=$ $\widehat{h}_{0}>(\pi / 2) \sum_{k \neq 0}\left|\widehat{h}_{k}\right|$, then as shown in [2, proof of Lemma 1, p. 435] the function $F$ given by

$$
F(x, y)=\widehat{h}_{0}+\sum_{s \neq 0} \frac{2 \pi i\left(s \alpha+l_{s}\right)}{e^{2 \pi i\left(s \alpha+l_{s}\right)}-1} \widehat{h}_{s} e^{2 \pi i\left(s x+l_{s} y\right)},
$$

where $l_{s}$ is the integer nearest to $-s \alpha$, is analytic. Observe that

$$
\left|\frac{2 \pi i\left(s \alpha+l_{s}\right)}{e^{2 \pi i\left(s \alpha+l_{s}\right)}-1}\right| \leq \frac{\pi}{2}
$$

and hence

$$
F(x, y) \geq \widehat{F}_{0,0}-\sum_{(k, l) \neq(0,0)}\left|\widehat{F}_{k, l}\right| \geq \widehat{h}_{0}-\frac{\pi}{2} \sum_{s \neq 0}\left|\widehat{h}_{s}\right|>0 .
$$

We end up this section by the observation that there exists a universal $d$ (depending only on $A>1$ ) such that all functions $f$ obtained from the a.a.c.c.p. (and given by (18)) satisfy

$$
d>\frac{\pi}{2} \sum_{s \neq 0}\left|\widehat{f}_{s}\right| .
$$

Indeed, from the proof of Lemma 5 it follows that whenever $d>\pi /(A-1)$ then for each $f$ given by (18) the inequality (33) holds.

4. An uncountable family of analytic flows. Put $E=\{0,1\}^{\mathbb{N}}$. In this section we will construct an uncountable family of cocycles $\left\{\varphi_{\varepsilon}\right\}_{\varepsilon \in E}$ so that for some equivalence relation $\sim \subset E \times E$ the corresponding special flows over an irrational rotation $T x=x+\alpha$ are disjoint whenever $\varepsilon \not \varepsilon^{\prime}$ and isomorphic whenever $\varepsilon \sim \varepsilon^{\prime}$.

Define now a cocycle $\varphi_{\varepsilon}$ in the following way. Given $\varepsilon=\left(\varepsilon_{i}\right)_{i \geq 1} \in E$ put

$$
\varphi_{\varepsilon}=\sum_{i=1}^{\infty} \widetilde{\varphi}_{i},
$$

where $\widetilde{\varphi}_{i}=\varepsilon_{i} \varphi_{i}$ and for $\varepsilon_{i}=1$ we carry out the $i$ th step of the a.a.c.c.p. from Subsection 2.2. The uncountable family of a.a.c.c.p.'s is now realized over a common $\alpha$ (indeed, recall that in view of Remark 3 we can replace some of the $d_{k i}$ 's by 0 and the a.a.c.c.p. will be realized over the same $\alpha$ ). Using similar arguments to those proving (26) we get $\mu\left(\left\{x \in[0,1): \widetilde{\varphi}_{k}^{\left(b_{k} q_{2 n_{k}}\right)}(x) \neq\right.\right.$ $\left.\left.\varphi_{\varepsilon}^{\left(b_{k} q_{2 n_{k}}\right)}(x)\right\}\right) \rightarrow 0$ as $k \rightarrow \infty$. Therefore

$$
\left(\varphi_{\varepsilon}^{\left(b_{k} q_{2 n_{k}}\right)}\right)_{*} \mu \rightarrow \nu \quad \text { as } k \rightarrow \infty, k \in\left\{i: \varepsilon_{i}=1\right\}
$$


if $\#\left\{i: \varepsilon_{i}=1\right\}=\infty$, and

$$
\left(\varphi_{\varepsilon}^{\left(b_{k} q_{2 n_{k}}\right)}\right)_{*} \mu \rightarrow \delta_{0} \quad \text { as } k \rightarrow \infty, k \in\left\{i: \varepsilon_{i}=0\right\}
$$

if $\#\left\{i: \varepsilon_{i}=0\right\}=\infty$.

Define $\sim \subset E \times E$ by putting

$$
\varepsilon \sim \varepsilon^{\prime} \quad \text { whenever } \varepsilon_{i}=\varepsilon_{i}^{\prime} \text { for } i \text { large enough. }
$$

Assume now that $\varepsilon \not \varepsilon^{\prime}$. So we can assume that the set $\left\{i \geq 1: \varepsilon_{i}=1\right.$, $\left.\varepsilon_{i}^{\prime}=0\right\}$ is infinite. Take a positive $d$ so that $\tilde{\varphi}_{\varepsilon}=\varphi_{\varepsilon}+d>0$ and $\widetilde{\varphi}_{\varepsilon^{\prime}}=$ $\varphi_{\varepsilon^{\prime}}+d>0$. In view of Proposition 2,

$$
\left(T^{\widetilde{\varphi}_{\varepsilon}}\right)_{d b_{k} q_{2 n_{k}}} \rightarrow \int_{\mathbb{R}}\left(T^{\widetilde{\varphi}_{\varepsilon}}\right)_{-t} d \nu(t), \quad\left(T^{\widetilde{\varphi}_{\varepsilon^{\prime}}}\right)_{d b_{k} q_{2 n_{k}}} \rightarrow \int_{\mathbb{R}}\left(T^{\widetilde{\varphi}_{\varepsilon^{\prime}}}\right)_{-t} d \delta_{0}(t),
$$

when $\varepsilon_{k}=1, \varepsilon_{k}^{\prime}=0$ and $k \rightarrow \infty$.

By Lemma 3 the special flows $\left(T_{t}^{\widetilde{\varphi}_{\varepsilon}}\right)_{t \in \mathbb{R}}$ and $\left(T_{t}^{\widetilde{\varphi}_{\varepsilon^{\prime}}}\right)_{t \in \mathbb{R}}$ are spectrally disjoint, hence they are disjoint in the sense of Furstenberg. Note that if $\varepsilon \sim \varepsilon^{\prime}$ then $\varphi_{\varepsilon}$ and $\varphi_{\varepsilon^{\prime}}$ are cohomologous and hence the corresponding special flows are isomorphic.

REMARK 5. Now, the argument used to prove (33) and the fact that $|\varphi| \leq 1$ imply that whenever $d>\max (1, \pi /(A-1))$,

$$
\varphi_{\varepsilon}+d>0 \quad \text { and } \quad d>\frac{\pi}{2} \sum_{s \neq 0}\left|\left(f_{\varepsilon}\right)_{s}^{\wedge}\right|
$$

for each $\varepsilon \in E$. Notice that the second inequality immediately implies that $f_{\varepsilon}+d>0$ for each $\varepsilon \in E$.

Put now $\tilde{f}_{\varepsilon}=f_{\varepsilon}+d$ and $\widetilde{f}_{\varepsilon^{\prime}}=f_{\varepsilon^{\prime}}+d$ and observe that the corresponding special flows also have the property that they are isomorphic if $\varepsilon \sim \varepsilon^{\prime}$ and disjoint (even spectrally disjoint) if $\varepsilon \not \varepsilon^{\prime}$.

REMARK 6. In view of Section 3 and Remark 5 for every (analytic) function $\tilde{f}_{\varepsilon}=f_{\varepsilon}+d$ we obtain an analytic function $F_{\varepsilon}:[0,1)^{2} \rightarrow \mathbb{R}_{+}$such that the special flow $T^{\tilde{f}_{\varepsilon}}$ and the flow $\mathcal{T}^{F_{\varepsilon}}$ coming from (29) are isomorphic. Remembering that the function $F_{\varepsilon}$ is given by $(30)$ we deduce that for all $\varepsilon \in E$,

$$
F_{\varepsilon}(x, y)>d-\frac{\pi}{A-1}>0
$$

On the other hand, from (30) and (31) we get, for all $(x, y) \in[0,1)^{2}$ and for all $\varepsilon \in E$,

$$
\left|F_{\varepsilon}(x, y)\right| \leq 2 d
$$

From now on we fix $d>\max (1, \pi /(A-1))$. 
REMARK 7. Observe that the family $\left\{\mathcal{T}^{F_{\varepsilon}}: \varepsilon \in E\right\}$ of analytic flows on $[0,1)^{2}$ obtained as above has the property that $\mathcal{T}^{F_{\varepsilon}}$ and $\mathcal{T}^{F_{\varepsilon^{\prime}}}$ are isomorphic whenever $\varepsilon \sim \varepsilon^{\prime}$, and disjoint (in fact, spectrally disjoint) for $\varepsilon \not \varepsilon^{\prime}$.

5. Non-existence of a countable set of Borel invariants in the class of flows on $\mathbb{R}^{2} / \mathbb{Z}^{2}$. The aim of this section is to show that a consequence of the results from the previous section is that it is not possible to find a countable complete set of Borel invariants for the measure-theoretic classification in the class of flows coming from (29). Such flows in general do not preserve Lebesgue measure, but they are non-singular with respect to it. Therefore we first recall some basic facts about topology of non-singular flows.

For a standard probability Borel space $(X, \mathcal{B}, \lambda)$ (we assume that $X$ is a compact space with a metric $\varrho$ ) consider the space $\widetilde{\operatorname{Aut}}(X, \mathcal{B}, \lambda)$ of all invertible non-singular automorphisms on $X$, i.e. $S \in \widetilde{\operatorname{Aut}}(X, \mathcal{B}, \lambda)$ if $S$ is invertible, bimeasurable and the measures $\lambda$ and $\lambda_{S}$ are equivalent, where $\lambda_{S}(A)=\lambda(S A)$ for every Borel set $A \subset X$.

We now define a Polish topology in $\widetilde{\operatorname{Aut}}(X, \mathcal{B}, \lambda)$ (see [23] for details).

For $S \in \widetilde{\operatorname{Aut}}(X, \mathcal{B}, \lambda)$ define

$$
\widetilde{U}_{S} f=\frac{d \lambda_{S}}{d \lambda} f \circ S, \quad f \in L^{1}(X, \mathcal{B}, \lambda) .
$$

We then observe that $\widetilde{U}_{S}$ belongs to the set Iso $\left(L^{1}(X, \lambda)\right)$ of invertible isometries of $L^{1}(X, \mathcal{B}, \lambda)$. Then by the Lamperti theorem the map

$$
\widetilde{\operatorname{Aut}}(X, \mathcal{B}, \lambda) \ni S \mapsto \widetilde{U}_{S} \in \operatorname{Iso}\left(L^{1}(X, \lambda)\right)
$$

is injective. On $\operatorname{Iso}\left(L^{1}(X, \lambda)\right)$ we consider the strong operator topology.

We endow $\widetilde{\operatorname{Aut}}(X, \mathcal{B}, \lambda)$ with a topology which makes the map (37) a homeomorphism. This topology is metrisable with a (complete) metric given by

$$
d(S, T)=\sum_{n=1}^{\infty} 2^{-n}\left(\left\|\widetilde{U}_{S} f_{n}-\widetilde{U}_{T} f_{n}\right\|_{1}+\left\|\widetilde{U}_{S^{-1}} f_{n}-\widetilde{U}_{T^{-1}} f_{n}\right\|_{1}\right),
$$

where $\left\{f_{n}^{\prime}\right\}_{n \geq 1}$ is a dense family in $L^{1}(X, \mathcal{B}, \lambda)$ and $f_{n}=f_{n}^{\prime} /\left\|f_{n}^{\prime}\right\|$.

REMARK 8. Assume that $S$ preserves the measure $F d \lambda$, where $F: X \rightarrow$ $\mathbb{R}_{+}$is measurable and in $L^{1}(\lambda)$. Observe that this is equivalent to

$$
F \circ S d \lambda_{S}=F d \lambda \text {. }
$$

Indeed, putting $\mu=F d \lambda$ we have

$$
\mu(A)=\mu(S A)=\int_{S A} F d \lambda=\int_{X} \chi_{A} \circ S^{-1} \cdot F d \lambda=\int_{A} F \circ S d \lambda_{S} .
$$


It follows that in the continuous case to show the convergence in the metric $d$ it is enough to show the uniform convergence of both: automorphisms and densities. Indeed, assume that $F_{n} \rightarrow F>0, F_{n}, F$ continuous and $S_{n} \rightarrow S$, $S_{n}^{-1} \rightarrow S^{-1}$ (uniformly) and that for each $n \in \mathbb{N}, S_{n}$ preserves the measure $F_{n} d \lambda$ (which by (38) is equivalent to the condition $d \lambda_{S_{n}} / d \lambda=F_{n} / F_{n} \circ S_{n}$ ). Then $F_{n} / F_{n} \circ S_{n} \rightarrow F / F \circ S$ uniformly and hence $d\left(S_{n}, S\right) \rightarrow 0$, as $n \rightarrow \infty$.

Put now $X=[0,1)^{2}, \mathcal{B}=\mathcal{B}\left([0,1)^{2}\right)$ and let $\lambda$ stand for Lebesgue measure on $[0,1)^{2}$. Denote by $\mathcal{N} \mathcal{F}\left([0,1)^{2}, \lambda\right)$ the space of all flows non-singular with respect to $\lambda$, that is, for each flow $\mathcal{T}=\left(T_{t}\right)_{t \in \mathbb{R}} \in \mathcal{N} \mathcal{F}\left([0,1)^{2}, \lambda\right)$ and all $t \in \mathbb{R}$, we have $T_{t} \in \widetilde{\operatorname{Aut}}\left([0,1)^{2}, \mathcal{B}\left([0,1)^{2}\right), \lambda\right)$. Moreover, we require that the unitary representation $t \mapsto \widetilde{\widetilde{U}}_{T_{t}}$ given by $\widetilde{\widetilde{U}}_{T_{t}} f=\sqrt{d \lambda_{T_{t}} / d \lambda} \cdot f \circ T_{t}$, $f \in L^{2}\left([0,1)^{2}, \lambda\right)$, is strongly continuous. The space $\mathcal{N} \mathcal{F}\left([0,1)^{2}, \lambda\right)$ with the metric

$$
D(\mathcal{T}, \mathcal{S})=\sup _{t \in[0,1]} d\left(T_{t}, S_{t}\right)
$$

becomes a Polish space (see [26] for the measure preserving case).

Consider now the subspace $\mathcal{N F}^{\prime}\left([0,1)^{2}, \lambda\right) \subset \mathcal{N} \mathcal{F}\left([0,1)^{2}, \lambda\right)$ of all flows arising from the systems of differential equations of the form (29) where $F \in C^{1}\left([0,1)^{2}\right)$. Denote by $\mathcal{N F}^{\omega}\left([0,1)^{2}, \lambda\right)$ the space (contained in $\left.\mathcal{N F}^{\prime}\left([0,1)^{2}, \lambda\right)\right)$ of flows arising from $(29)$ with $F$ analytic. On $\mathcal{N} \mathcal{F}^{\prime}\left([0,1)^{2}, \lambda\right)$ and $\mathcal{N}^{\omega}\left([0,1)^{2}, \lambda\right)$ we consider the restriction of the metric $D$.

REMARK 9. Observe that each element $\mathcal{T}^{F}=\left(T_{t}^{F}\right)_{t} \in \mathcal{N F}^{\prime}\left([0,1)^{2}, \lambda\right)$ preserves a measure equivalent to Lebesgue measure $\lambda$, namely $F d \lambda$. Hence using similar arguments to those in Remark 8 we find that the convergence in this space follows from the uniform convergence of densities and time $t$ automorphisms. In other words, to obtain $D\left(\mathcal{T}^{F_{n}}, \mathcal{T}^{F}\right) \rightarrow 0$ as $n \rightarrow \infty$ it suffices to show that $T_{t}^{F_{n}}(x, y) \rightarrow T_{t}^{F}(x, y)$ uniformly with respect to $(t, x, y)$, $t \in[-1,1]$ and that $F_{n}(x, y) \rightarrow F(x, y)$ uniformly with respect to $(x, y)$.

Recall that $\alpha$ defines the common rotation for the whole family $\left\{F_{\varepsilon}\right\}_{\varepsilon \in E}$ obtained in Section 4. Without changing the notation let us go back to the construction in which we have obtained the family $\left\{\mathcal{T}^{F_{\varepsilon}}: \varepsilon \in E\right\}$ of (analytic) flows on $[0,1)^{2}$. Consider the map

$$
E=\{0,1\}^{\mathbb{N}} \ni \varepsilon \stackrel{\Theta}{\longmapsto} \mathcal{T}^{F_{\varepsilon}} \in \mathcal{N F}^{\omega}\left([0,1)^{2}, \lambda\right) .
$$

We equip $E=\{0,1\}^{\mathbb{N}}$ with the product topology (which is metrisable with, for instance, the product metric $\left.d_{E}\left(\left(x_{n}\right),\left(y_{n}\right)\right)=\sum_{k \geq 1} 2^{-k}\left|x_{k}-y_{k}\right|\right)$ so that $E$ becomes a compact topological space.

Put

$$
\mathcal{A}=\Theta(E)=\left\{\mathcal{T}^{F_{\varepsilon}}: \varepsilon \in E\right\} .
$$

The main result of this section is the following. 
Theorem 2. The set $\mathcal{A} \subset \mathcal{N F}^{\omega}\left([0,1)^{2}, \lambda\right)$ is a Borel subset of $\mathcal{N F}\left([0,1)^{2}, \lambda\right)$. Moreover, if $\widetilde{\varphi}: \mathcal{A} \rightarrow \mathbb{R}^{\infty}$ is an arbitrary Borel function such that $\widetilde{\varphi}(\mathcal{T})=\widetilde{\varphi}(\mathcal{S})$ whenever $\mathcal{T} \in \mathcal{A}$ and $\mathcal{S} \in \mathcal{A}$ are isomorphic, then there exist spectrally disjoint flows $\mathcal{T}^{\prime} \in \mathcal{A}$ and $\mathcal{S}^{\prime} \in \mathcal{A}$ such that $\widetilde{\varphi}\left(\mathcal{T}^{\prime}\right)=\widetilde{\varphi}\left(\mathcal{S}^{\prime}\right)$.

From Theorem 2 we immediately get the following.

Corollary 3. There does not exist a Borel map $\widetilde{\varphi}: \mathcal{N F}\left([0,1)^{2}, \lambda\right) \rightarrow$ $\mathbb{R}^{\infty}$ which is constant on the (spectral) isomorphism classes and simultaneously takes different values for (spectrally) non-isomorphic arguments.

In other words, it is not possible to find a countable complete set of Borel invariants in $\mathcal{N F}\left([0,1)^{2}, \lambda\right)$ for the problem of (spectral) isomorphism.

Proof of Theorem 2. It is sufficient to show that

(i) $\Theta$ is continuous (hence $\mathcal{A}$ is compact),

(ii) if $\widetilde{\varphi}: \mathcal{A} \rightarrow \mathbb{R}^{\infty}$ is a Borel function constant on isomorphism classes, then there exist non-isomorphic flows $\mathcal{T}^{F_{\varepsilon}}$ and $\mathcal{T}^{F_{\varepsilon^{\prime}}}$ such that $\widetilde{\varphi}\left(\mathcal{T}^{F_{\varepsilon}}\right)$ $=\widetilde{\varphi}\left(\mathcal{T}^{F_{\varepsilon^{\prime}}}\right)$.

First observe that (ii) follows from the 0-1 Kolmogorov law (by considering properties of $\widetilde{\varphi} \circ \Theta$ ).

It remains to show (i). Assume that $\varepsilon_{n} \rightarrow \varepsilon$, as $n \rightarrow \infty$. In view of Remark 9 , to show that $D\left(\mathcal{T}^{F_{\varepsilon_{n}}}, \mathcal{T}^{F_{\varepsilon}}\right) \rightarrow 0$ it is enough to show that

$$
T_{t}^{F_{\varepsilon_{n}}}(x, y) \rightarrow T_{t}^{F_{\varepsilon}}(x, y)
$$

uniformly with respect to $(t, x, y), t \in[-1,1]$ and that

$$
F_{\varepsilon_{n}}(x, y) \rightarrow F_{\varepsilon}(x, y)
$$

uniformly with respect to $(x, y)$.

To see (40) we will prove the continuity of the map

$$
E \ni \varepsilon \mapsto F_{\varepsilon} \in C\left([0,1)^{2}\right) .
$$

This can be replaced by a stronger condition that the mapping $E \ni \varepsilon \mapsto$ $\widehat{F}_{\varepsilon} \in l^{1}\left(\mathbb{Z}^{2}\right)$ is continuous. Furthermore in view of (30) and (31) it is enough to show the continuity of the map $E \ni \varepsilon \mapsto \widehat{f}_{\varepsilon} \in l^{1}(\mathbb{Z})$, and this latter condition is proved in much the same way as Lemma 5 .

To prove (39) recall that a differential equation on $[0,1)^{2}$ may be lifted to an equation on $\mathbb{R}^{2}$ simply by periodic extension. For $\varepsilon \in E$ consider then a differential equation of the form (29), that is,

$$
\frac{d \mathcal{X}}{d t}=\Gamma_{\varepsilon}(\mathcal{X})
$$

where $\mathcal{X}=(x, y), \Gamma_{\varepsilon}: \mathbb{R}^{2} \rightarrow \mathbb{R}^{2}$ and $\Gamma_{\varepsilon}(\mathcal{X})=\left(\alpha / F_{\varepsilon}(\mathcal{X}), 1 / F_{\varepsilon}(\mathcal{X})\right)$. 
Since $F_{\varepsilon}$ is analytic, it is Lipschitz (with a constant $L_{\varepsilon}$ ), which implies that $\Gamma_{\varepsilon}$ is also Lipschitz with the constant

$$
\widetilde{L}_{\varepsilon}=\frac{\sqrt{\alpha^{2}+1}}{\left(d-\frac{\pi}{A-1}\right)^{2}} L_{\varepsilon}
$$

i.e. for all $(x, y) \in \mathbb{R}^{2}$ and $\left(x^{\prime}, y^{\prime}\right) \in \mathbb{R}^{2}$,

$$
\left\|\Gamma_{\varepsilon}(x, y)-\Gamma_{\varepsilon}\left(x^{\prime}, y^{\prime}\right)\right\|_{\mathbb{R}^{2}} \leq \widetilde{L}_{\varepsilon}\left\|(x, y)-\left(x^{\prime}, y^{\prime}\right)\right\|_{\mathbb{R}^{2}}
$$

Then the equation (41) has a solution defined for all $t \in \mathbb{R}$, of the form

$$
\bar{T}_{t}^{F_{\varepsilon}}(x, y)=(x, y)+\int_{0}^{t} \Gamma_{\varepsilon}(x(\tau), y(\tau)) d \tau
$$

for each $(x, y) \in \mathbb{R}^{2}$. Observe that for the flows given by solutions of (41) and (29) we have

$$
\left\|T_{t}^{F_{\varepsilon}}(x, y)-T_{t}^{F_{\varepsilon^{\prime}}}(x, y)\right\|_{[0,1)^{2}} \leq\left\|\bar{T}_{t}^{F_{\varepsilon}}(x, y)-\bar{T}_{t}^{F_{\varepsilon^{\prime}}}(x, y)\right\|_{\mathbb{R}^{2}},
$$

because $T_{t}^{F_{\varepsilon}}=\bar{\pi} \circ \bar{T}_{t}^{F_{\varepsilon}}$, where $\bar{\pi}: \mathbb{R}^{2} \rightarrow[0,1)^{2}$ is the natural quotient map.

Now from the Gronwall inequality it follows that

$$
\left\|\bar{T}_{t}^{F_{\varepsilon^{\prime}}}(x, y)-\bar{T}_{t}^{F_{\varepsilon}}(x, y)\right\|_{\mathbb{R}^{2}} \leq \sqrt{\alpha^{2}+1}\left(d-\frac{\pi}{A-1}\right)^{-2}\left\|F_{\varepsilon}-F_{\varepsilon^{\prime}}\right\|_{\infty} e^{\widetilde{L}_{\varepsilon^{\prime}}} .
$$

Indeed, observe that

$$
\begin{aligned}
& \left\|\bar{T}_{t}^{F_{\varepsilon^{\prime}}}(x, y)-\bar{T}_{t}^{F_{\varepsilon}}(x, y)\right\|_{\mathbb{R}^{2}}=\left\|\int_{0}^{t}\left(\Gamma_{\varepsilon^{\prime}}\left(\bar{T}_{\tau}^{F_{\varepsilon^{\prime}}}(x, y)\right)-\Gamma_{\varepsilon}\left(\bar{T}_{\tau}^{F_{\varepsilon}}(x, y)\right)\right) d \tau\right\|_{\mathbb{R}^{2}} \\
& \leq \int_{0}^{t}\left\|\Gamma_{\varepsilon^{\prime}}\left(\bar{T}_{\tau}^{F_{\varepsilon^{\prime}}}(x, y)\right)-\Gamma_{\varepsilon^{\prime}}\left(\bar{T}_{\tau}^{F_{\varepsilon}}(x, y)\right)\right\|_{\mathbb{R}^{2}} d \tau \\
& \quad+\int_{0}^{t}\left\|\Gamma_{\varepsilon^{\prime}}\left(\bar{T}_{\tau}^{F_{\varepsilon}}(x, y)\right)-\Gamma_{\varepsilon}\left(\bar{T}_{\tau}^{F_{\varepsilon}}(x, y)\right)\right\|_{\mathbb{R}^{2}} d \tau \\
& \leq \int_{0}^{t} \widetilde{L}_{\varepsilon^{\prime}}\left\|\bar{T}_{\tau}^{F_{\varepsilon^{\prime}}}(x, y)-\bar{T}_{\tau}^{F_{\varepsilon}}(x, y)\right\|_{\mathbb{R}^{2}} d \tau+t \sqrt{\alpha^{2}+1}\left\|\frac{1}{F_{\varepsilon^{\prime}}}-\frac{1}{F_{\varepsilon}}\right\|_{\infty} \\
& \leq \int_{0}^{t} \widetilde{L}_{\varepsilon^{\prime}}\left\|\bar{T}_{\tau}^{F_{\varepsilon^{\prime}}}(x, y)-\bar{T}_{\tau}^{F_{\varepsilon}}(x, y)\right\|_{\mathbb{R}^{2}} d \tau+\sqrt{\alpha^{2}+1}\left(d-\frac{\pi}{A-1}\right)^{-2}\left\|F_{\varepsilon}-F_{\varepsilon^{\prime}}\right\|_{\infty} .
\end{aligned}
$$

Hence (43) follows. And from (43) we immediately get (39).

6. Weak closure of time $t$ automorphisms of an analytic flow. In this section we will construct a weakly mixing flow $T^{\widetilde{\varphi}}$ with the following property: 
(44) for each $P \in \mathcal{P}(\mathbb{R})$ the Markov operator $\int_{\mathbb{R}} T_{t}^{\widetilde{\varphi}} d P(t)$ belongs to the weak closure of $\left\{T_{t}^{\widetilde{\varphi}}: t \in \mathbb{R}\right\}$.

This flow will be isomorphic to an analytic flow on $[0,1)^{2}$. By (9) to prove (44) we only need to consider $P$ running over a dense subset of $\mathcal{P}(\mathbb{R})$, for example measures of the form $\sum_{j=1}^{Q} B_{j} \delta_{r_{j}}$, where $r_{j} \in \mathbb{Q}, B_{j} \in \mathbb{Q}_{+}$and $\sum_{j=1}^{Q} B_{j}=1$. Our goal is to construct a flow $T^{\widetilde{\varphi}}$ satisfying:

(45) For each $\widetilde{M} \in \mathbb{N}_{+}, s \in \mathbb{N}, r_{j} \in \mathbb{Q}, A_{j} \in \mathbb{Q}_{+}, j=1, \ldots, \widetilde{M}, \widetilde{A}:=$ $\sum_{j=1}^{\widetilde{M}} A_{j}<1$, the Markov operator $\int_{\mathbb{R}} T_{t}^{\widetilde{\varphi}} d P(t)$ with

$$
P=\sum_{j=1}^{\widetilde{M}} A_{j} \delta_{r_{j}}+\frac{1-\widetilde{A}}{2}\left(\delta_{s-\frac{2}{1-\widetilde{A}} \sum_{j=1}^{\widetilde{M}} r_{j} A_{j}}+\delta_{-s}\right)
$$

belongs to the weak closure of the set $\left\{T_{t}^{\widetilde{\varphi}}: t \in \mathbb{R}\right\}$.

The construction goes as follows. Consider the set

$$
\begin{aligned}
\left\{\left(\left(r_{1}, \ldots, r_{\widetilde{M}}\right),\left(A_{1}, \ldots, A_{\widetilde{M}}\right), s\right): r_{j} \in \mathbb{Q}, A_{j} \in \mathbb{Q}_{+},\right. & \\
& \left.\sum_{j=1}^{\widetilde{M}} A_{j}<1, s \in \mathbb{N}, \widetilde{M} \in \mathbb{N}_{+}\right\} .
\end{aligned}
$$

Note that this set is countable and denote by $\left(U_{k}\right)_{k \geq 1}$ a sequence

$$
U_{k}=\left(\left(r_{1 k}, \ldots, r_{\widetilde{M}_{k} k}\right),\left(A_{1 k}, \ldots, A_{\widetilde{M}_{k} k}\right), s_{k}\right)
$$

in which for each element $\left(\left(r_{1}, \ldots, r_{\widetilde{M}}\right),\left(A_{1}, \ldots, A_{\widetilde{M}}\right), s\right)$ of the above set we have $\#\left\{k \in \mathbb{N}_{+}: U_{k}=\left(\left(r_{1}, \ldots, r_{\widetilde{M}}\right),\left(A_{1}, \ldots, A_{\widetilde{M}}\right), s\right)\right\}=\infty$. We need more notation: $A_{j k}=g_{j k} / f_{j k}\left(g_{j k}, f_{j k} \in \mathbb{N}_{+}\right), j=1, \ldots, \widetilde{M}_{k}, \widetilde{A}_{k}=\sum_{j=1}^{\widetilde{M}_{k}} A_{j k}$, $\left(1-\widetilde{A}_{k}\right) / 2=g_{\widetilde{A}_{k}} / f_{\widetilde{A}_{k}}, g_{\widetilde{A}_{k}}, f_{\widetilde{A}_{k}} \in \mathbb{N}_{+}, \widetilde{m}_{k}=\operatorname{LCM}\left(f_{1 k}, \ldots, f_{\widetilde{M}_{k} k}, f_{\widetilde{A}_{k}}\right)$, $N_{j k}=\min \left\{m \in \mathbb{N}_{+}:\left|r_{j k} / m\right|<1\right\}$,

$$
\widetilde{N}_{k}=\min \left\{m \in \mathbb{N}_{+}:\left|\frac{s_{k}-\frac{2}{1-\widetilde{A}_{k}} \sum_{j=1}^{\widetilde{M}_{k}} r_{j k} A_{j k}}{m}\right|<1\right\} \text {. }
$$

We will now carry out an a.a.c.c.p. with extra conditions on the parameters; namely, we require that $D_{k} \leq 1$ (see Remark 3) and that

$$
M_{k}>m_{k}\left(N_{1 k} A_{1 k}+\cdots+N_{\widetilde{M}_{k} k} A_{\widetilde{M}_{k} k}+\frac{1-\widetilde{A}_{k}}{2}\left(\widetilde{N}_{k}+s_{k}+1\right)\right),
$$

where $m_{k}=c_{k} \widetilde{m}_{k}, c_{k} \in \mathbb{N}, c_{k} \rightarrow \infty$ and

$$
\left(\sum_{i=1}^{k-1} M_{i}\right) \sqrt{\frac{2}{m_{k}}} \leq \text { const. }
$$


Observe that

$$
N_{1 k} A_{1 k}+\cdots+N_{\widetilde{M}_{k} k} A_{\widetilde{M}_{k} k}+\frac{1-\widetilde{A}_{k}}{2}\left(\widetilde{N}_{k}+s_{k}+1\right) \geq 1,
$$

hence $m_{k} \varepsilon_{k} \rightarrow 0$ as $k \rightarrow \infty$ (recall that $\sum_{k=1}^{\infty} \sqrt{\varepsilon_{k}} \cdot M_{k}<\infty$ ). As $m_{k} \rightarrow \infty$, we have $M_{k} \rightarrow \infty$. Let $\alpha$ be an irrational over which the a.a.c.c.p. is carried out. We require additionally that $a_{2 n_{k}+1}$ is a multiple of $m_{k}$, more precisely

$$
a_{2 n_{k}+1}=m_{k} b_{k},
$$

where $b_{k} \geq 1$ for all $k \geq 1$. Recall that

$$
\lambda_{k} M_{k}<\frac{\varepsilon_{k}}{2 q_{2 n_{k}}}
$$

so $\lambda_{k} M_{k}=\mathrm{o}\left(\mu\left(I_{k}\right)\right)$. Denote by $\xi_{1}, \ldots, \xi_{m_{k}}$ the consecutive subintervals of $I_{k}$ of length $\mu\left(I_{k}\right) / m_{k}$. This means that any such subinterval consists of $b_{k}$ intervals $J_{t}^{k}$. We now define $\varphi_{k}$ as follows. If $\varepsilon_{k} m_{k}>2 / 3$ we put arbitrary $d_{k i}$ according to general rules for a.a.c.c.p. Assume now that $\varepsilon_{k} m_{k} \leq 2 / 3$. Notice that

$$
\lambda_{k} M_{k} \leq \frac{3}{2} \cdot \frac{\varepsilon_{k}}{3 q_{2 n_{k}}} \leq \frac{3}{2} \varepsilon_{k} \mu\left(I_{k}\right) \leq \frac{1}{m_{k}} \mu\left(I_{k}\right)=\left|\xi_{i}\right|,
$$

$i=1, \ldots, m_{k}$. Given $j=1, \ldots, \widetilde{M}_{k}$, in each interval $\xi_{\left(A_{1 k}+\cdots+A_{j-1, k}\right) m_{k}+v}$, $v=1, \ldots, A_{j k} m_{k}$, we choose $N_{j k}$ consecutive intervals $\omega_{k i}$ so that the interval $\bigcup \omega_{k i}$ is placed "centrally", i.e. in the middle of $\xi_{\left(A_{1 k}+\cdots+A_{j-1, k}\right) m_{k}+v}$. In the central interval $J_{s_{k, i}^{k}} \subset \omega_{k i}$ we put the value $r_{j k} / N_{j k}$. Analogously in each interval $\xi_{\widetilde{A}_{k} m_{k}+v}, v=1, \ldots,\left(1-\widetilde{A}_{k}\right) m_{k} / 2$, we select "centrally" $\widetilde{N}_{k}$ consecutive intervals $\omega_{k i}$ and in the central subintervals we put the value

$$
\frac{s_{k}-\frac{2}{1-\widetilde{A}_{k}} \sum_{j=1}^{\widetilde{M}_{k}} r_{j k} A_{j k}}{\widetilde{N}_{k}} \text {. }
$$

Finally, in each of $\xi_{\left(\widetilde{A}_{k}+1\right) m_{k} / 2+v}, v=1, \ldots,\left(1-\widetilde{A}_{k}\right) m_{k} / 2$, we select "centrally" $s_{k}+1$ consecutive $\omega_{k i}$ on which in the central subintervals we put $-s_{k} /\left(s_{k}+1\right)$. In order to complete the a.a.c.c.p. we choose $\omega_{k i}$ arbitrarily and put $d_{k i}=0$ for the remaining

$$
i \in\left\{m_{k}\left(\sum_{i=1}^{\widetilde{M}_{k}} N_{i k} A_{i k}+\frac{1-\widetilde{A}_{k}}{2}\left(\widetilde{N}_{k}+s_{k}+1\right)\right)+1, \ldots, M_{k}\right\}
$$

which is possible by (49). Notice that $\sum_{i=1}^{M_{k}} d_{k i}=0$.

Now, we fix $U=\left(\left(r_{1}, \ldots, r_{\widetilde{M}}\right),\left(A_{1}, \ldots, A_{\widetilde{M}}\right), s\right)$ and consider only $k$ such that $U_{k}=U$. First notice that $m_{k} \varepsilon_{k}<2 / 3$ for sufficiently large such $k$. Moreover,

$$
\frac{\max \left(N_{1}, \ldots, N_{\widetilde{M}}, \widetilde{N}_{k}, s+1\right) \lambda_{k} m_{k}}{\mu\left(I_{k}\right)} \rightarrow 0
$$


as $k \rightarrow \infty$ with $U_{k}=U\left(\widetilde{N}_{k}=\widetilde{N}\right)$ by (48). Thus

$$
\left(\varphi_{k}^{\left(b_{k} q_{2 n_{k}}\right)}(x)\right)_{*} \mu \rightarrow \sum_{j=1}^{\widetilde{M}} A_{j} \delta_{r_{j}}+\frac{1-\widetilde{A}}{2}\left(\delta_{s-\frac{2}{1-\widetilde{A}} \sum_{j=1}^{\widetilde{M}} r_{j} A_{j}}+\delta_{-s}\right)
$$

as $k \rightarrow \infty$ with $U_{k}=U$.

Now using similar arguments to those showing (26) and the fact that $m_{k} \rightarrow \infty$ as $k \rightarrow \infty$, we obtain

$$
\mu\left(\left\{x \in[0,1): \varphi_{k}^{\left(b_{k} q_{2 n_{k}}\right)}(x) \neq \varphi^{\left(b_{k} q_{2 n_{k}}\right)}(x)\right\}\right) \rightarrow 0
$$

as $k \rightarrow \infty$. Thus

$$
\left(\varphi^{\left(b_{k} q_{2 n_{k}}\right)}(x)\right)_{*} \mu \rightarrow \sum_{j=1}^{\widetilde{M}} A_{j} \delta_{r_{j}}+\frac{1-\widetilde{A}}{2}\left(\delta_{s-\frac{2}{1-\widetilde{A}} \sum_{j=1}^{\widetilde{M}} r_{j} A_{j}}+\delta_{-s}\right)
$$

as $k \rightarrow \infty$ with $U_{k}=U$.

Obviously $|\varphi(x)| \leq 1$ for $x \in[0,1)$. Take now a constant $d$ such that $\widetilde{\varphi}=\varphi+d>0$. Using similar arguments to those in Subsection 2.2 (with (47) instead of (16)) we will show that along the subsequence $\left(b_{k} q_{2 n_{k}}\right)$ where $k \in\left\{i: U_{i}=U=\left(\left(r_{1}, \ldots, r_{\widetilde{M}}\right),\left(A_{1}, \ldots, A_{\widetilde{M}}\right), s\right)\right\},\left\|\varphi^{\left(b_{k} q_{2 n_{k}}\right)}\right\|_{L^{2}}<$ const. Indeed, the only thing to notice is that for all $x \in[0,1)$ and $k \in\left\{i: U_{i}=\right.$ $\left.U=\left(\left(r_{1}, \ldots, r_{\widetilde{M}}\right),\left(A_{1}, \ldots, A_{\widetilde{M}}\right), s\right)\right\}$,

$$
\begin{aligned}
& \left|\varphi_{k}^{\left(b_{k} q_{2 n_{k}}\right)}(x)\right| \\
& \quad \leq \max \left\{e+f: e, f \in\left\{\left|r_{1}\right|, \ldots,\left|r_{\widetilde{M}}\right|,\left|s-\frac{2}{1-\widetilde{A}} \sum_{j=1}^{\widetilde{M}} r_{j} A_{j}\right|,|s|\right\}\right\} \\
& \quad=\text { const. }
\end{aligned}
$$

The construction of $T^{\widetilde{\varphi}}$ is complete, and by Proposition 2, (45) has been proved.

In this way we see that for each $k \geq 1$ there exists an increasing sequence $\left(q_{n}^{(k)}\right)$ such that

$$
T_{d q_{n}^{(k)}}^{\widetilde{\varphi}} \rightarrow \int_{\mathbb{R}} T_{-t}^{\widetilde{\varphi}} d P_{k}(t)
$$

where $\left\{P_{k}\right\}$ is a dense subset in $\mathcal{P}(\mathbb{R})$.

Now in view of (9) we get (44):

Corollary 4. For each measure $P \in \mathcal{P}(\mathbb{R})$ there exists a sequence $\left(q_{n}^{(P)}\right) \subseteq \mathbb{N}$ such that

$$
T_{d q_{n}^{(P)}}^{\widetilde{\varphi}} \rightarrow \int_{\mathbb{R}} T_{-t}^{\widetilde{\varphi}} d P(t)
$$

Assume now that $d \in \mathbb{N}$. Then we obtain the following. 
Corollary 5. For each measure $P \in \mathcal{P}(\mathbb{Z})$ there exists a sequence $\left(q_{n}^{(P)}\right) \subseteq \mathbb{N}$ such that

$$
\left(T_{1}^{\widetilde{\varphi}}\right)^{q_{n}^{(P)}} \rightarrow \sum_{j=-\infty}^{\infty} P(j)\left(T_{1}^{\widetilde{\varphi}}\right)^{-j}
$$

REMARK 10. Recall (see e.g. [15]) that a flow $\left(S_{t}\right)_{t \in \mathbb{R}}$ is called $\kappa$-weakly mixing $(0 \leq \kappa \leq 1)$ if there exists a sequence $\left(t_{n}\right)$ with $t_{n} \rightarrow \infty$ such that

$$
S_{t_{n}} \rightarrow \kappa \mathrm{Id}+(1-\kappa) \int
$$

By taking $P_{n}=\frac{1}{4} \delta_{-n}+\frac{1}{2} \delta_{0}+\frac{1}{4} \delta_{n}$ and considering $n=n_{k}$ so that $T_{n_{k}}^{\widetilde{\varphi}} \rightarrow \int$ (which is justified by weak mixing of $T^{\widetilde{\varphi}}$ ), by Corollary $5, \frac{1}{2} \operatorname{Id}+\frac{1}{2} \int$ belongs to the weak closure of the set $\left\{\left(T^{\widetilde{\varphi}}\right)_{t}: t \in \mathbb{R}\right\}$, i.e. $T^{\widetilde{\varphi}}$ is $\frac{1}{2}$-weakly mixing. This implies mutual singularity of convolutions of maximal spectral type (see [15], [24], [27]).

However, using a result of O. N. Ageev (see [1]) we can obtain much more. If $T^{\widetilde{\varphi}}$ satisfies the assumptions of Corollary 5 then the maximal spectral

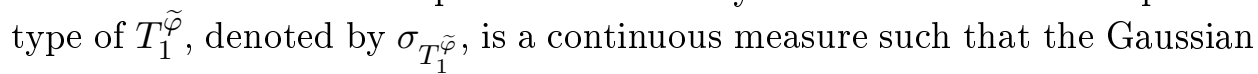
system $S$ determined by it has a simple spectrum. In particular, convolutions of the reduced maximal spectral type of $T_{1}^{\widetilde{\varphi}}$ are mutually singular. Since $T_{1}^{\widetilde{\varphi}}$ is disjoint from all ELF-automorphisms (see [3]), it is disjoint from all Gaussian automorphisms. Hence the Gaussian system $S$ has the GAG property, but it cannot satisfy the Foiaş-Strătilă theorem (indeed, if a system satisfies the Foiaş-Strătilă theorem then it is Gaussian; see [20] for details).

\section{References}

[1] O. N. Ageev, On ergodic transformations with homogeneous spectrum, J. Dynam. Control Systems 5 (1999), 149-152.

[2] I. P. Cornfeld, S. W. Fomin and Ya. G. Sinai, Ergodic Theory, Springer, Berlin, 1982.

[3] Y. Derriennic, K. Frączek, M. Lemańczyk and F. Parreau, Ergodic automorphisms whose weak closure of off-diagonal measures consists of ergodic self-joinings, preprint.

[4] B. Fayad and A. Windsor, A dichotomy between discrete and continuous spectrum for a class of special flows over rotations, J. Modern Dynamics 1 (2007), 107-122.

[5] J. Feldman, Borel structures and invariants for measurable transformations, Proc. Amer. Math. Soc. 46 (1974), 383-394.

[6] M. Foreman, A descriptive view of ergodic theory, in: Descriptive Set Theory and Dynamical Systems, London Math. Soc. Lecture Note Ser. 277, Cambridge Univ. Press, 2000.

[7] K. Frączek, On ergodicity of some cylinder flows, Fund. Math. 163 (2000), 117-130.

[8] K. Frączek and M. Lemańczyk, On symmetric logarithm and some old examples in smooth ergodic theory, Fund. Math. 180 (2003), 241-255. 
[9] K. Frączek and M. Lemańczyk, A class of special flows over irrational rotations which is disjoint from mixing flows, Ergodic Theory Dynam. Systems 24 (2004), 1083-1095.

[10] -, - On disjointness properties of some smooth flows, Fund. Math. 185 (2005), $117-142$.

[11] - - - On mild mixing of special flows over irrational rotations under piecewise smooth functions, Ergodic Theory Dynam. Systems 26 (2006), 719-738.

[12] H. Furstenberg, Disjointness in ergodic theory, minimal sets and diophantine approximation, Math. Systems Theory 1 (1967), 1-49.

[13] F. Hahn and W. Parry, Some characteristic properties of dynamical systems with quasi-discrete spectra, Math. Systems Theory 2 (1968), 179-190.

[14] A. del Junco and M. Lemańczyk, Generic spectral properties of measure-preserving maps and applications, Proc. Amer. Math. Soc. 115 (1992), 725-736.

[15] A. B. Katok, Constructions in Ergodic Theory, unpublished lecture notes.

[16] L. Kuipers and H. Niederreiter, Uniform Distribution of Sequences, Wiley, New York, 1974.

[17] J. Kwiatkowski, M. Lemańczyk and D. Rudolph, A class of real cocycles having an analytic coboundary modification, Israel J. Math. 87 (1994), 337-360.

[18] M. Lemańczyk and E. Lesigne, Ergodicity of Rokhlin cocycles, J. Anal. Math. 85 (2001), 43-86.

[19] M. Lemańczyk and F. Parreau, Rokhlin extensions and lifting disjointness, Ergodic Theory Dynam. Systems 23 (2003), 1525-1550.

[20] M. Lemańczyk, F. Parreau and J.-P. Thouvenot, Gaussian automorphisms whose ergodic self-joinings are Gaussian, Fund. Math. 164 (2000), 253-293.

[21] M. Lemańczyk, F. Parreau and D. Volný, Ergodic properties of real cocycles and pseudo-homogeneous Banach spaces, Trans. Amer. Math. Soc. 348 (1996), 49194938.

[22] P. Liardet and D. Volný, Sums of continuous and differentiable functions in dynamical systems, Israel J. Math. 98 (1997), 29-60.

[23] M. G. Nadkarni, Spectral Theory of Dynamical Systems, Birkhäuser, Basel, 1998.

[24] A. Prikhod'ko and V. V. Ryzhikov, Disjointness of the convolutions for Chacon's automorphism, Colloq. Math. 84/85 (2000), 67-74.

[25] E. Roy, Mesures de Poisson, infinie divisibilité et propriétés ergodiques, $\mathrm{PhD}$ thesis.

[26] T. de la Rue and J. de Sam Lazaro, Une transformation générique peut être insérée dans un flot, Ann. Inst. H. Poincaré Probab. Statist. 39 (2003), 121-134.

[27] A. M. Stepin, On properties of spectra of ergodic dynamical systems with locally compact time, Dokl. Akad. Nauk SSSR 169 (1966), 773-776 (in Russian).

[28] D. Volný, Constructions of smooth and analytic cocycles over irrational circle rotations, Comment. Math. Univ. Carolin. 36 (1995), 745-764.

[29] R. J. Zimmer, Ergodic Theory and Semisimple Groups, Birkhäuser, Boston, 1984.

Faculty of Mathematics and Computer Science

Nicolaus Copernicus University

Chopina 12/18, 87-100 Toruń, Poland

E-mail: mlem@mat.uni.torun.pl mwysokin@mat.uni.torun.pl

Received 11 May 2005;

in revised form 28 June 2006 